\title{
Hydropod: An on-board deployed acoustic-visual device for propeller cavitation and noise investigations
}

\author{
Batuhan Aktas 1, *, Mehmet Atlar'1, Spyros Leivadaros ${ }^{2}$, Noriyuki Sasaki ${ }^{1}$, Patrick \\ Fitzsimmons $^{1}$
}

${ }^{1}$ Naval Architecture, Ocean \& Marine Engineering, University of Strathclyde

Glasgow G4 0LZ, UK

${ }^{2}$ School of Marine Science and Technology, Newcastle University, Newcastle upon Tyne

NE1 7RU, UK

Corresponding Author:

*Batuhan Aktas, batuhan.aktas@strath.ac.uk

Henry Dyer Building

100 Montrose Street

Naval Architecture, Ocean \& Marine Engineering,

University of Strathclyde

Glasgow G4 0LZ, UK

Tel: +44(0) 1415484094 


\begin{abstract}
:
Conducting noise trials with big merchant vessels could constitute serious economical and time losses for the ship operators. This study aims to introduce an experimental acoustic-visual device enabling economical and cost effective noise trials in full-scale. By means of hydrophones and a high-definition, wide-angle underwater camera appropriately fitted on a customised submerged device, called "Hydropod"; noise emission and dynamics of the cavitation phenomenon are investigated on a research catamaran. The previously conducted noise trials following the international standards with an off-board hydrophone array are utilised for the validation of the adopted approach. The comparisons between the Hydropod measurements and conventional noise trial measurement results have shown promising correlations, except the self-noise hump present in the noise spectra of the Hydropod measurements. Furthermore, by taking advantage of the replacement of the conventional propellers of the catamaran with a set of New Profile Technology (NPT) propellers, additional trials were conducted using the Hydropod. This enabled interpretation of the relative performance of both sets of propellers in terms of acoustics and cavitation extent. The NPT propellers, in this case, excelled with their superiority compared to the conventional propellers over the cavitation extent and resulting acoustic emissions.
\end{abstract}

Keywords: Underwater Radiated Noise; Propeller Cavitation Noise; Experimental Hydrodynamics; Full-scale noise trials 


\section{Introduction}

The technological advancements over the last century have led to significant developments in the maritime industry. The development of the steam engines as a driving force for the ship propulsion and further gains in efficiency with the diesel engines has resulted in a big boom being experienced by the industry. These developments were furthered by the hydrodynamics research. Particularly, those regarding the cavitation resulted in further enhancement of the efficiency of marine transportation. This consequently revealed itself as an increasing trend amongst the commercial shipping in terms of the engine size, number of ships in service and the operating speed. In return, the transportation of a good from one place to another became relatively cheaper and easier which created the concept of globalisation.

However, with the rising trend of the commercial shipping volume, various emissions produced by the vessels have also started to increase [1]. This trend has resulted in significant elevation of various emissions by the seagoing vessels. In order to ensure sustainable shipping, various anthropogenic impacts have been targeted by the rising environmental awareness. Whilst initial focus of international organisations was on greenhouse gas output of the maritime transportation, recently underwater noise created by commercial ships has been targeted, due to its potential for impact on the marine fauna. 'Although there is not a complete understanding of the impact of underwater noise from commercial shipping on marine fauna, ambient noise measurements indicate a potentially dangerous tendency [2]. The consequences of this trend experienced by the world fleet have been observed to impact the low frequency ambient noise levels as it is the dominant noise source in this frequency region, as identified by Wenz (1962). Namely, the measurements made in the Pacific Ocean indicate a $3 \mathrm{~dB}$ increase per decade (or 
$10 \mathrm{~dB}$ per half century) in terms of measured background noise levels, as summarized in Figure 1 by Frisk, based on the compiled historical ambient noise data [4-6].

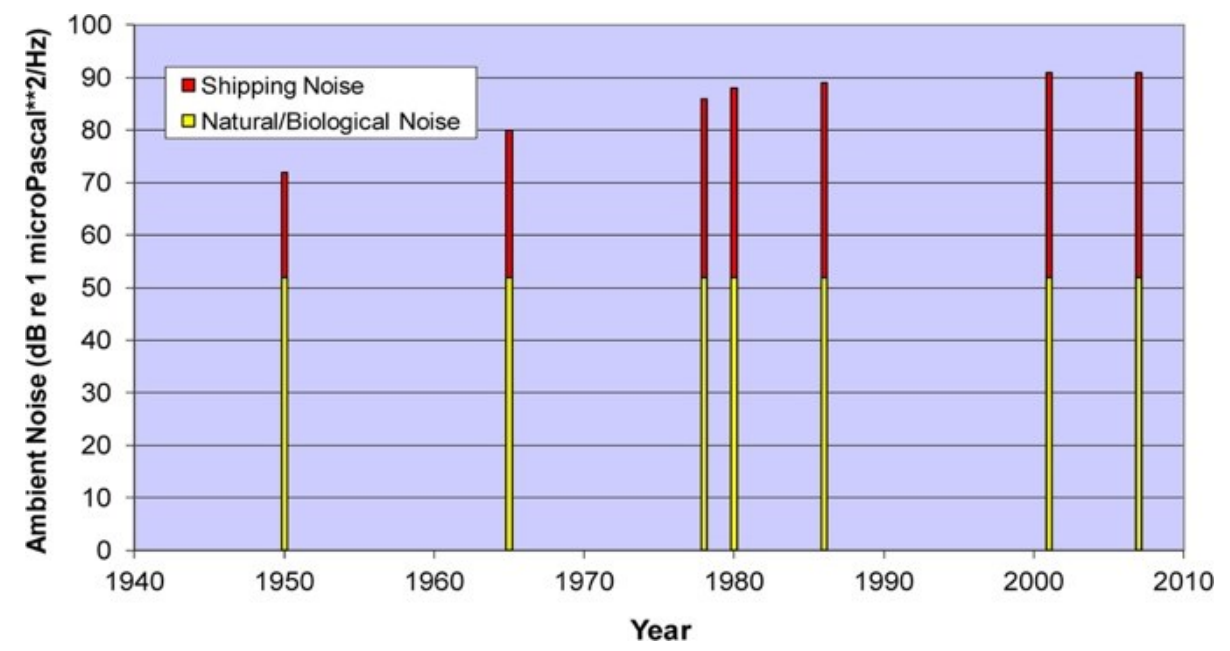

Figure 1 Historical ambient noise levels in $25-50 \mathrm{~Hz}$ indicating the increasing trend of shipping contribution

(Reproduced from Frisk, 2012)

Thus, URN has been targeted by environmentalists due to its potential threat to marine fauna when it is also borne in mind that the low frequency region is utilised by marine mammals for various fundamental living activities [7]. Consequently, this results in underwater noise pollution in the low-frequency range that may have an adverse effect on the survivability of marine mammals by disrupting their behaviours or causing distress $[8,9]$.

The issue has been flagged up during various sessions of International Maritime Organization Marine Environment Protection Committee meetings [10,11]. The rising environmental awareness over the potential destruction of the marine fauna has resulted in a surge of interest in the underwater noise produced by commercial shipping. Moreover, the Marine Strategy Framework Directive (MSFD) determined the commercial shipping to be a dominant factor in the rising ambient noise levels [12]. In order to maintain the Good Environmental Status (GES), 
proposed mitigation is to be introduced by 2020 and monitoring of the European seas by 2018 [13].

The developments in international scale resulted in potential resuscitation of voluntary silent class notations $[14,15]$ to be adopted for the mitigation and regulation purposes. However, the assessment procedure of underwater noise for a commercial ship is cumbersome and costly. The amount of time required for conducting the ISO standard underwater noise trials can constitute a significant amount of time for a vessel off the trade. Whilst there are various standards available for conducting full-scale noise trials such as ANSI and ISO [16,17], the inherent nature of the underwater noise trials require change of course from the trade route and sailing to the trial site as well as time-consuming trial preparation. In case of a potential enforcement of limits on the radiated noise levels of a commercial vessel, current standards for noise survey may be deemed to be too costly in terms of economic losses.

Within this framework, this study presents an experimental method for underwater noise assessment of a vessel using a practical measurement device deployed and attached on-board of the vessel. The developed and engineered equipment is named 'Hydropod' and referred to with this name from here onwards. Hydropod measurements enable cost efficient and accurate noise assessment whilst requiring minimal time off the trade, less change of course of the route and significantly reduced preparation requirement. Furthermore, the introduced method also includes an underwater camera system enabling the observation of the cavitation experienced hence enhancing the understanding of the noise creating mechanisms. 
In order to evaluate the accuracy of the proposed measurement technique, previously conducted noise trials following the international standards [17] with an off-board hydrophone array, are utilised for the validation of the adopted approach. The comparisons between the Hydropod measurements and conventional noise trial measurement results have shown promising correlations, except the self-noise hump present in the noise spectra of the Hydropod measurements. Following this, taking advantage of the replacement of the original propellers of The Newcastle University Research Vessel "The Princess Royal" with a set of New Profile Technology (NPT) propellers, a second trial is conducted to appraise the relative cavitation and noise performance of either set of propellers.

This paper is structured to contain five sections. Following this introductory section, full-scale trials are discussed in depth in Section 2 including various crucial aspects such as target vessel “The Princess Royal”, details about the full scale conventional and NPT propellers, Hydropod design procedure and adopted trial methodology. Then, details of the noise measurements and analysis with Hydropod are presented together with the comparisons with conventional offboard measurements for justification of Hydropod measurements and evaluation of the hydroacoustic performance of NPT are given in Section 3. Cavitation observations with the old and NPT propellers are presented in Section 4. Finally, the conclusions drawn out of this study are presented in Section 5 .

\section{Full-Scale Trials}

The full-scale trials conducted with the Hydropod prototype had various vital elements. These have included target research vessel "The Princess Royal", two propeller sets (original conventional propellers and NPT propellers), the prototype Hydropod and the adopted trial 
methodology. These aspects are introduced and reviewed in detail within the following subsections.

\subsection{Research Vessel "The Princess Royal"}

Full-scale trials of the Hydropod were conducted using Newcastle University Research vessel "The Princess Royal". She is a displacement type of Deep-V catamaran, which was designed in-house and built locally, as described in detail by [18] and Figure 2 shows the views of the research vessel whilst, Table 1 displays the main particulars of "The Princess Royal”.
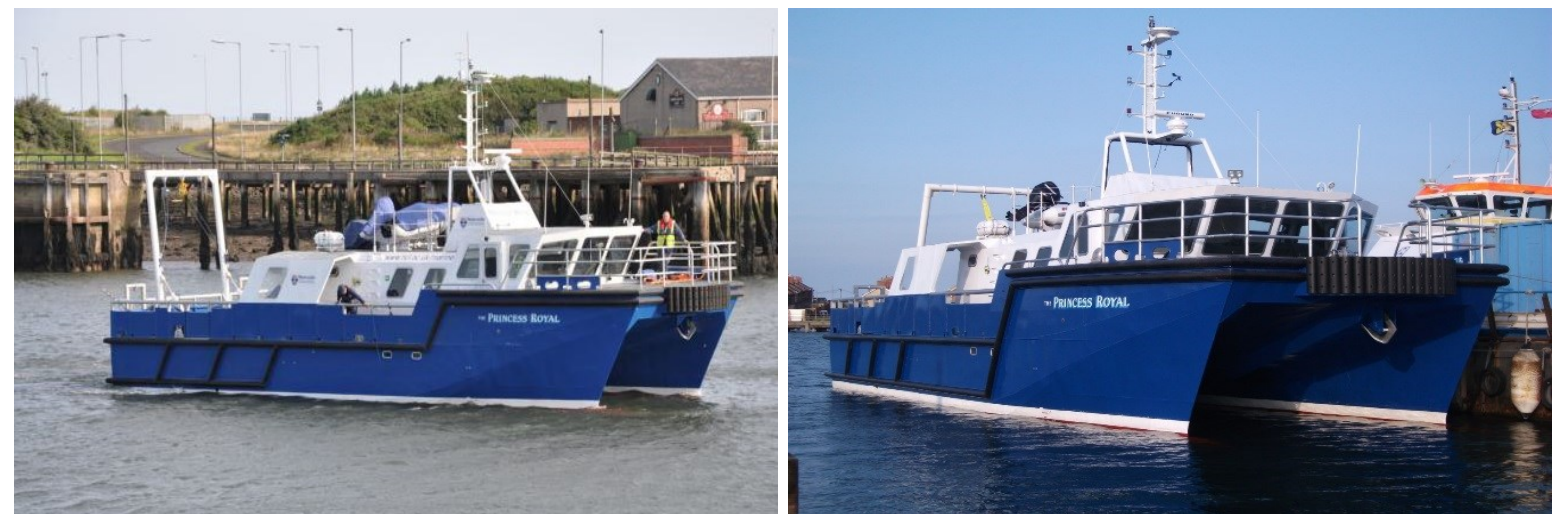

Figure 2 Views of Newcastle University's new research vessel, "The Princess Royal" 
Table 1 Main particulars of "The Princess Royal" as built

\begin{tabular}{|c|c|}
\hline Launching date \& place & 11 August 2011 \& Alnmaritec, Blyth (UK) \\
\hline Hullform & Displacement type Deep-V catamaran with symmetric \\
& demi-hulls \\
\hline Length overall & $18.88 \mathrm{~m}$. \\
\hline Length BP & $16.45 \mathrm{~m}$. \\
\hline Breadth Moulded & $7.03 \mathrm{~m}$. \\
\hline Breadth Extreme & $7.34 \mathrm{~m}$. \\
\hline Depth moulded & $3.18 \mathrm{~m}$. \\
\hline Demi-hulls separation (C $\mathbf{L}_{\mathbf{L}}$ to $\mathbf{C}_{\mathbf{L}}$ ) & $4.9 \mathrm{~m}$. \\
\hline Displacement (Lightship) & 36.94 tonnes \\
\hline Draught (Lightship) (Amid - FP - AP) & \\
\hline Deadweight data & 7.32 tonnes (Excluding 2 tonnes of deck cargo) \\
\hline
\end{tabular}

\subsection{Full-Scale Propellers: Conventional and NPT}

The Princess Royal has been designed in-house by staff and students in the School of Marine Science and Technology. The vessel is a marriage of the catamaran and deep-V hull form concepts and this combination provides the vessel with enhanced seakeeping, efficiency and speed. The data collected from a wide range of sea trials has proven the superior hydrodynamic performance of the vessel as expected. However, some severe tasks during her research activities involving trawling and bollard pull tasks have caused propeller deformations and the efficiency of the propellers was deteriorated. In addition, the vessel was launched with a weight greater than the planned design displacement and hence the propellers of the vessel were operating at off-design conditions. 
In order to address above problems, a new set of propellers were designed for the research vessel based on NPT (New Profile Technology) [19]. The design conditions of the new propellers were estimated by analysing the available full-scale data of the vessel performance with the old propellers for the nominal (i.e. not deteriorated) condition to determine the in service operating condition of the propeller. The speed and power curves were thus estimated accounting for the improvement in propeller efficiency obtained by the replacement of the existing propellers with the new ones. The design conditions of the new propeller are propitiously clearer than those used for the original propellers because of a wealth of Full Scale data hitherto collected by not only sea trials but also by in-service monitoring.

Table 2 shows the comparative principal particulars of the old and new propellers whilst Figure 3 illustrates the difference in pitch distributions between two propellers. It is very clear that the new propeller has approximately $20 \%$ less blade area in comparison to the original propellers and this was achieved by the new profile technology (NPT) design method. In spite of the lesser area the new design aimed for a smaller cavitation extent from $0.5 \mathrm{R}$ to tip where an extremely thick and large cavitation extent was observed with the original propellers. The maximum difference of the pitch angle $(\alpha)$ is $-1.3^{\circ}$ which appears to be around $0.9 \mathrm{R}$. 


\begin{tabular}{|c|c|c|}
\hline & Old & NPT \\
\hline Diameter (mm) & \multicolumn{2}{|c|}{750} \\
\hline Pitch Ratio & 0.8475 & 0.845 \\
\hline Exp. Area Ratio & 1.057 & 0.83 \\
\hline Blade Number & \multicolumn{2}{|c|}{5} \\
\hline Skew Angle (deg.) & 20 & 30 \\
\hline Forward Skew (deg) & 5 & 15 \\
\hline Boss Ratio & \multicolumn{2}{|c|}{0.18} \\
\hline t/c at 0.7R & 0.0392 & 0.0414 \\
\hline
\end{tabular}

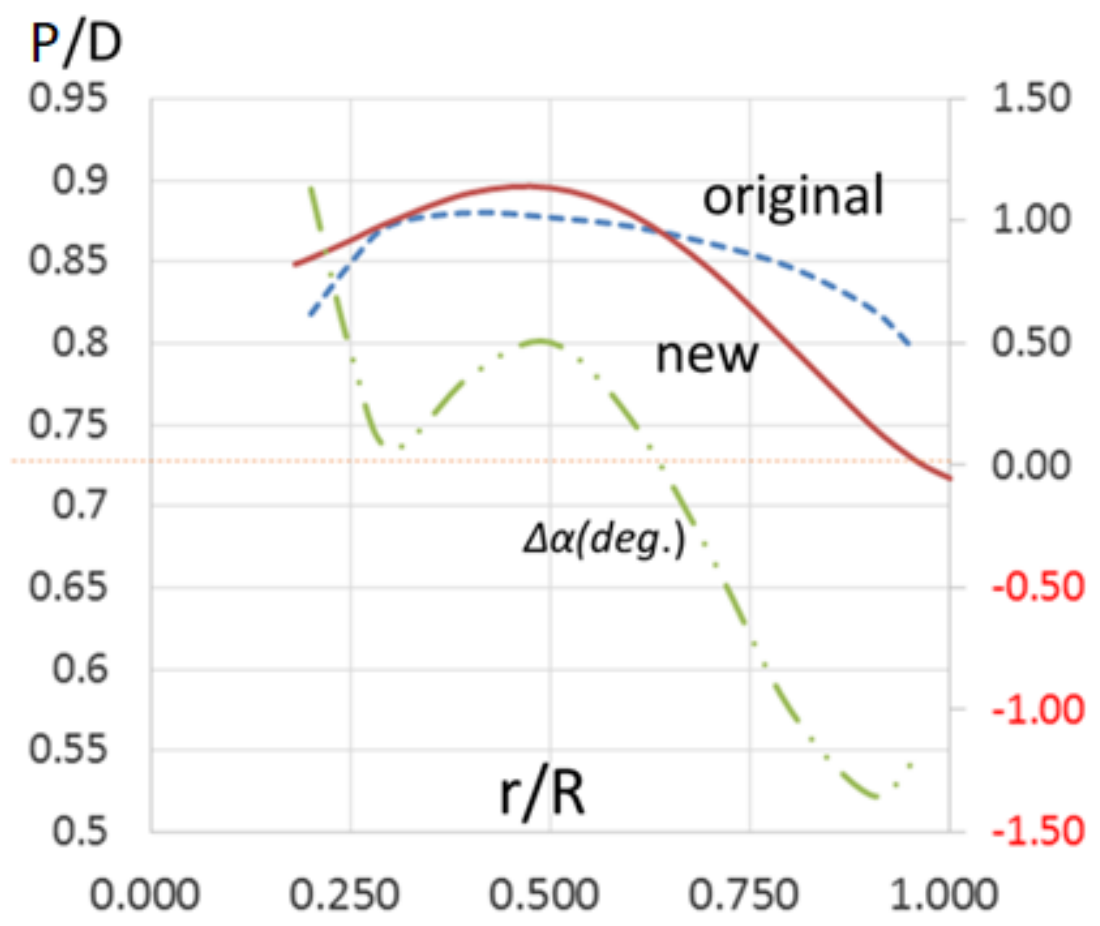

Figure 3 Difference of pitch ratio and pitch angles

Notwithstanding the same skew at tip (about 101mm) was applied to both existing propellers and new propellers, the skewness of the new propeller is more effective because the sweep angle is much larger as a result of a different skew line and blade counter. This skew line is also expected to be more effective for reducing the hull pressure fluctuations, but it requires 
more detailed analysis for strength design. Figure 4 show the old and new NPT propeller on the starboard shaft of The Princess Royal.

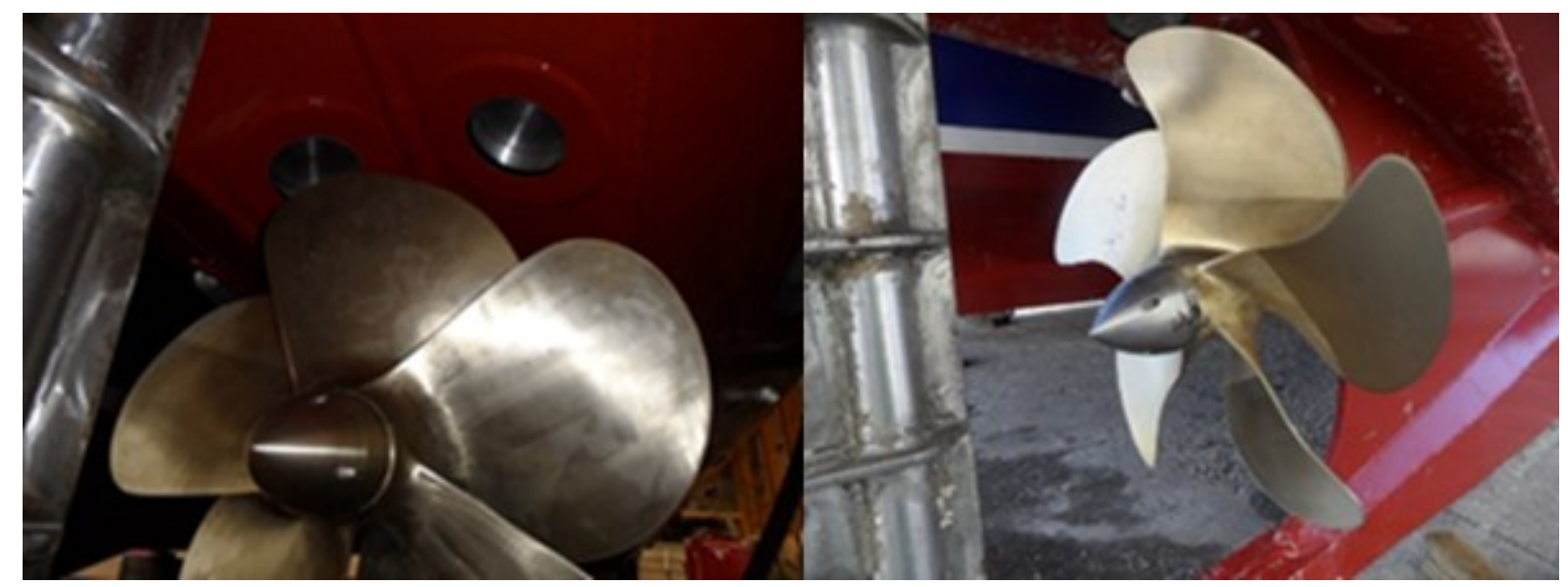

Figure 4 Conventional (Left) and NPT (Right) propeller of "The Princess Royal"

\subsection{Hydropod Design}

The idea of relating the full-scale propeller cavitation observations with accompanied acoustic data led to the design of a hydro-dynamically shaped device that is able to sustain induced pressures and forces generated from the forward speed of the vessel when she is underway. A symmetric, vertical glass fibre hydrofoil was designed, constructed and named "Hydropod". At the upper end, it was stiffly mounted on the vessel's hand rails, while at the lower end it was shaped into a convenient pod on which the camera and hydrophone were accommodated as shown in Figure 5 and Figure 6. Hydropod is developed to enable deployment from port, starboard or aft side of RV Princess Royal as demonstrated by Figure 7 for the starboard side. 

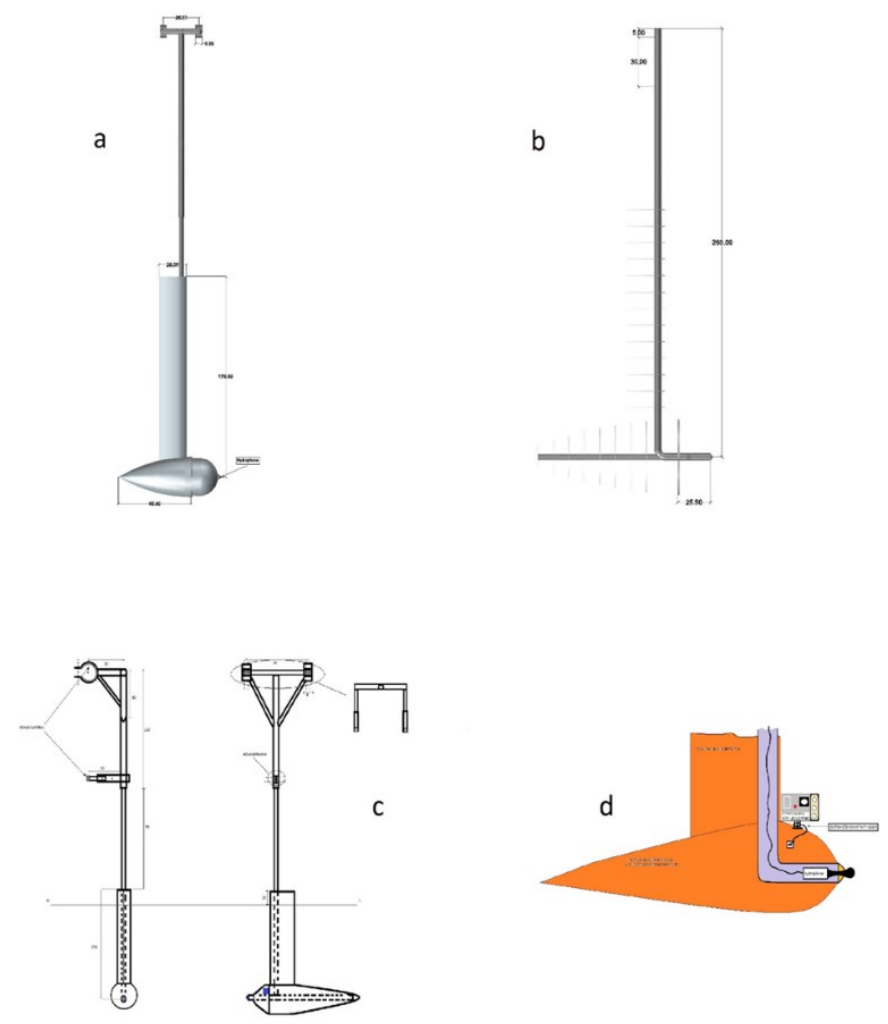

Figure 5 a) side view of the Hydropod together with the upper mounting part, b) x-ray view of the device (aluminium stiffening plates are visible), c) a preliminary sketch of entire structure, d) the form which finally adopted.

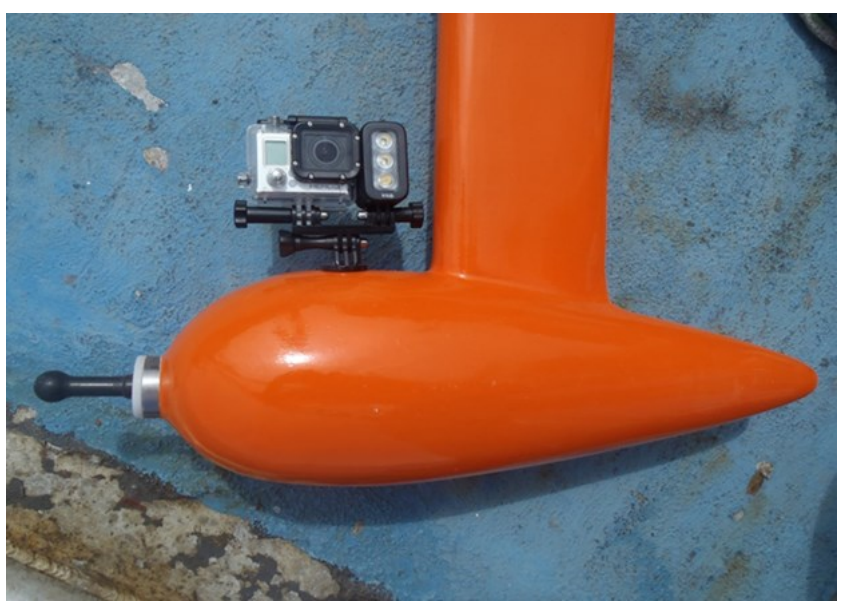

Figure 6 Hydrophone, camera and LED lighting attached to the Hydropod. 


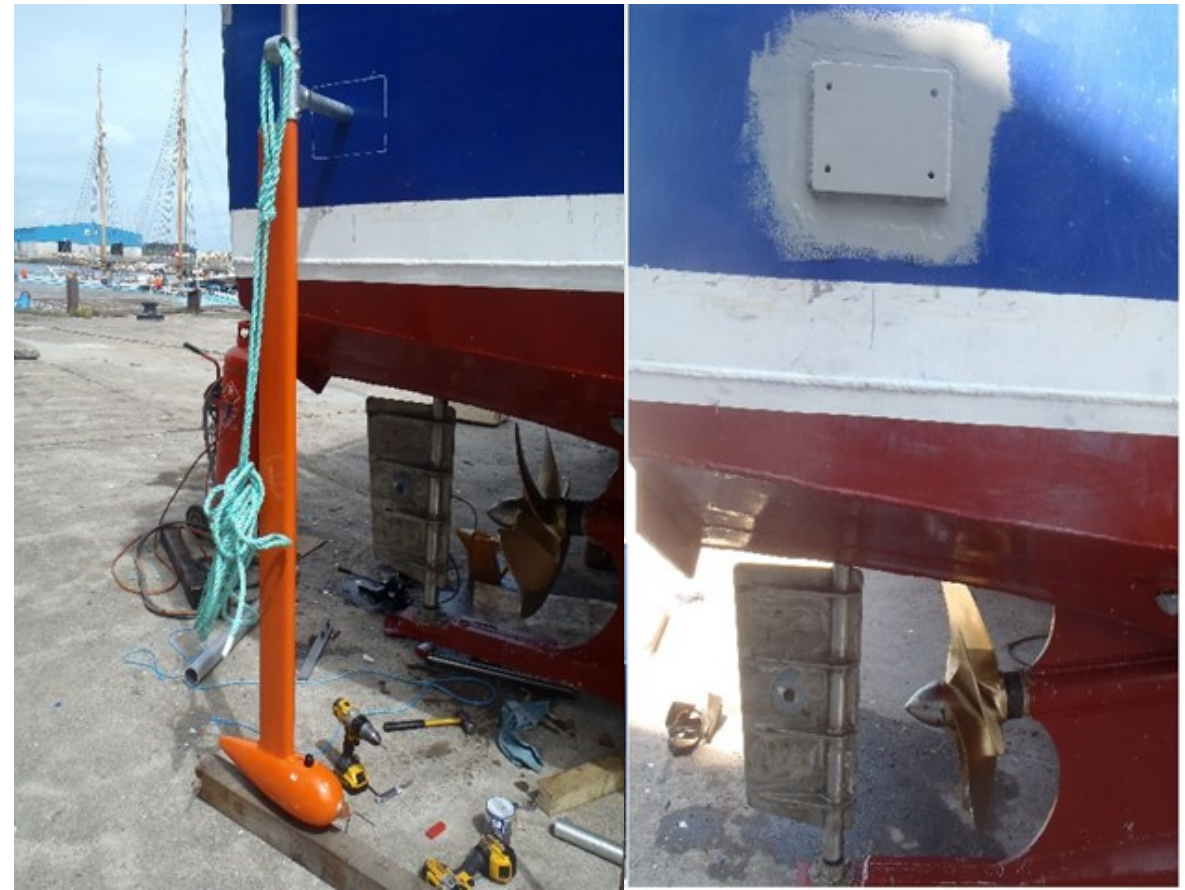

Figure 7 Attachment of the Hydropod to the vessel during drydock for underwater camera adjustment.

The inspiration for the Hydropod came from the strut type hyrophone installments in cavitation tunnels [20] as shown by Figure 8 . The general structure of this approach was adapted to be applied in full-scale for the first time. 


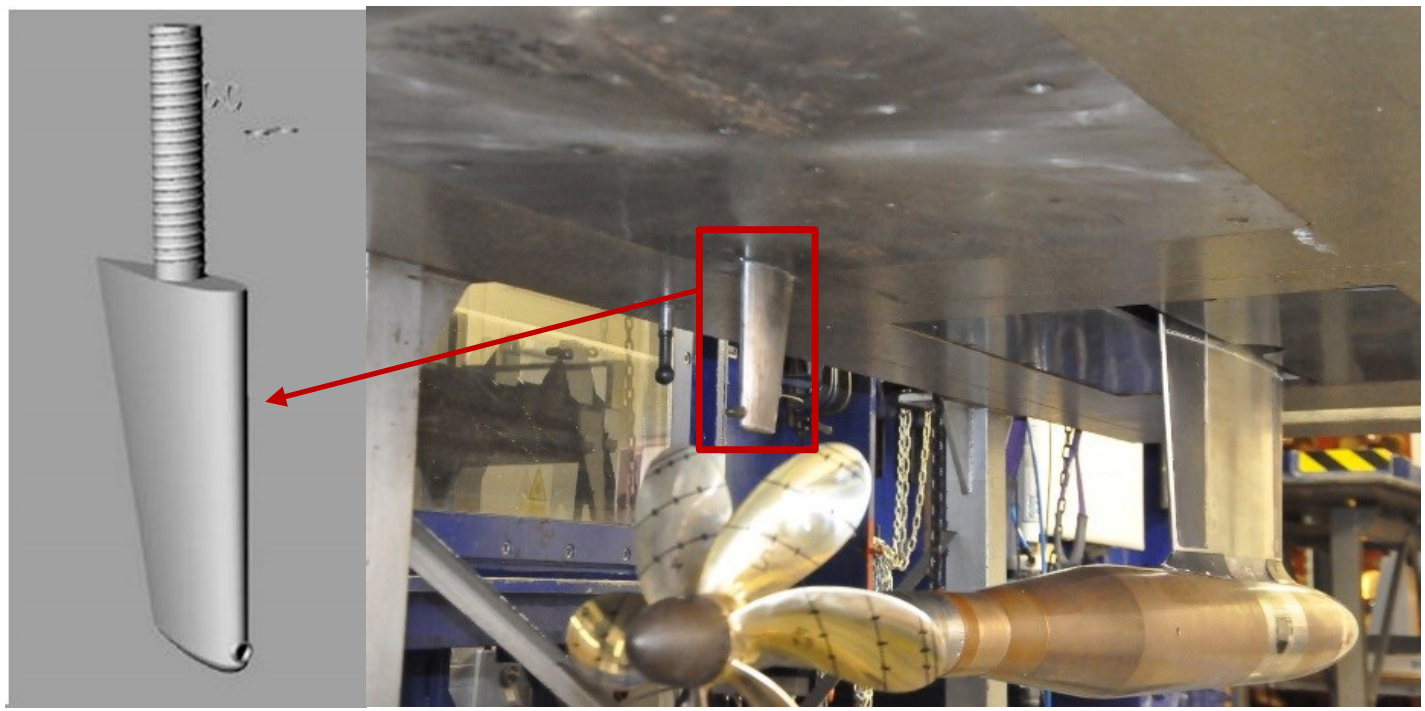

Figure 8 streamlined foil support to fit inside the cavitation tunnel test section for hydrophone attachment.

The present device is experimental and could be refined to be much smaller and to suit larger ships by being bolted onto a heavy duty flange on the hull plating. The device could be attached at any port and even moved to other locations while at sea in stationary by using a diver. The Hydropod concept maybe alternatively be modified to resemble a towing hydrophone array. This will require updating the current design by adding fins for stability while being towed and removing the strut that is used for attaching to the hand rails.

\subsection{Trial methodology}

The developed prototype also necessitated a dedicated noise trial procedure to be determined for Hydropod. After careful review of a number of standards and procedures that are produced for use of conventional off-board noise trials [13,14,16,17,21] and guidelines provided for execution of speed and power trials ([22]), a fusion method is established and adapted to use of Hydropod. 
Following ITTC (2012), trials were conducted with reasonably flat sea surface while the weather conditions were corresponding to Beaufort scale of 2 or less in order to minimise the impact of the added resistance on ship due to wind and wave, and rudder helm angle to be lower than 5 degrees while on run. The effect of current was minimised by conducting the runs with and against the current to eliminate the effect by averaging the data. Minimum water depth below the keel of the vessel was maintained at least $30 \mathrm{~m}$ at all times. The trial runs were conducted for north and south runs while the Hydropod located at the starboard and port of the vessel, totalling up to four individual runs for one operating condition. Unlike ISO, (2016), a Williamson turn for the reciprocal run was not made following the completion of one condition. Instead, the following condition was conducted on the same course due to Hydropod being less stable during manoeuvring.

Following the completion of the conditions in one direction (i.e. with the current), a Williamson turn was executed to bring the vessel to opposite course (i.e.,.against the current) for the reciprocal runs. This did not only enabled less disruption to the fixtures of the Hydropod but also allowed execution of the trials within shorter time periods to ensure sufficient battery time for cavitation observations. A fully charged battery would enable continuous recording for an hour, providing ample time for completion either starboard or port aspect measurements before replacing the battery of the underwater camera before deployment from the other aspect.

Ultimately, the full-scale trials were held in the North Sea by the beginning (15.06.2015) and the middle (23.07.2015) of summer 2015. The trials were conducted two nautical mile distance from the port of Blyth. The trials were carried out for four different engine RPM conditions. The choice of using engine RPM was made due to the ease of fixing the condition for the 
skipper of the vessel and RPM conditions were chosen based on the availability of the data from previous full-scale trials $[23,24]$. Namely, these conditions were chosen as 600 RPM, 900 RPM 1200 RPM and 1500 RPM. The propeller shaft rotational speed was lower due to the gearbox ratio of 1.75:1. The maximum speed was limited due to the concerns about the structural integrity of the Hydropod and its inherent design being prone to bend. Whilst, Hydropod performed exceptionally up to 1200 RPM, during the 1500 RPM with the old propeller, minor bending started to occur. This RPM condition was thus halted and has not been executed with the old propellers. During the drydocking period for the replacement of the propellers, significant improvements to the Hydropod fixture were also carried out, such as the addition of a lower supporting plate and additional supports. The trials were separated into two groups as North run and South run due to the current direction in the trial region. The runs were also repeated for starboard and port aspect by simple removal of the Hydropod and reattachment to the other side, which essentially takes a maximum of 30 minutes. With the initiation of the North run, each RPM condition was fixed for 5 minutes enabling sufficient time for the collection of noise data and cavitation observation recordings. Following the completion of the 5 minutes, subsequent RPM condition was set and run for 5 minutes. Following the completion of the North run for all four RPM conditions, the vessel was turned to south with a Williamson turn and same procedure was repeated for four RPM condition following the same method. The chart plotter data from the vessel deck was plotted and presented together with the accompanying vessel engine RPM against speed data is presented as shown in Figure 9. 


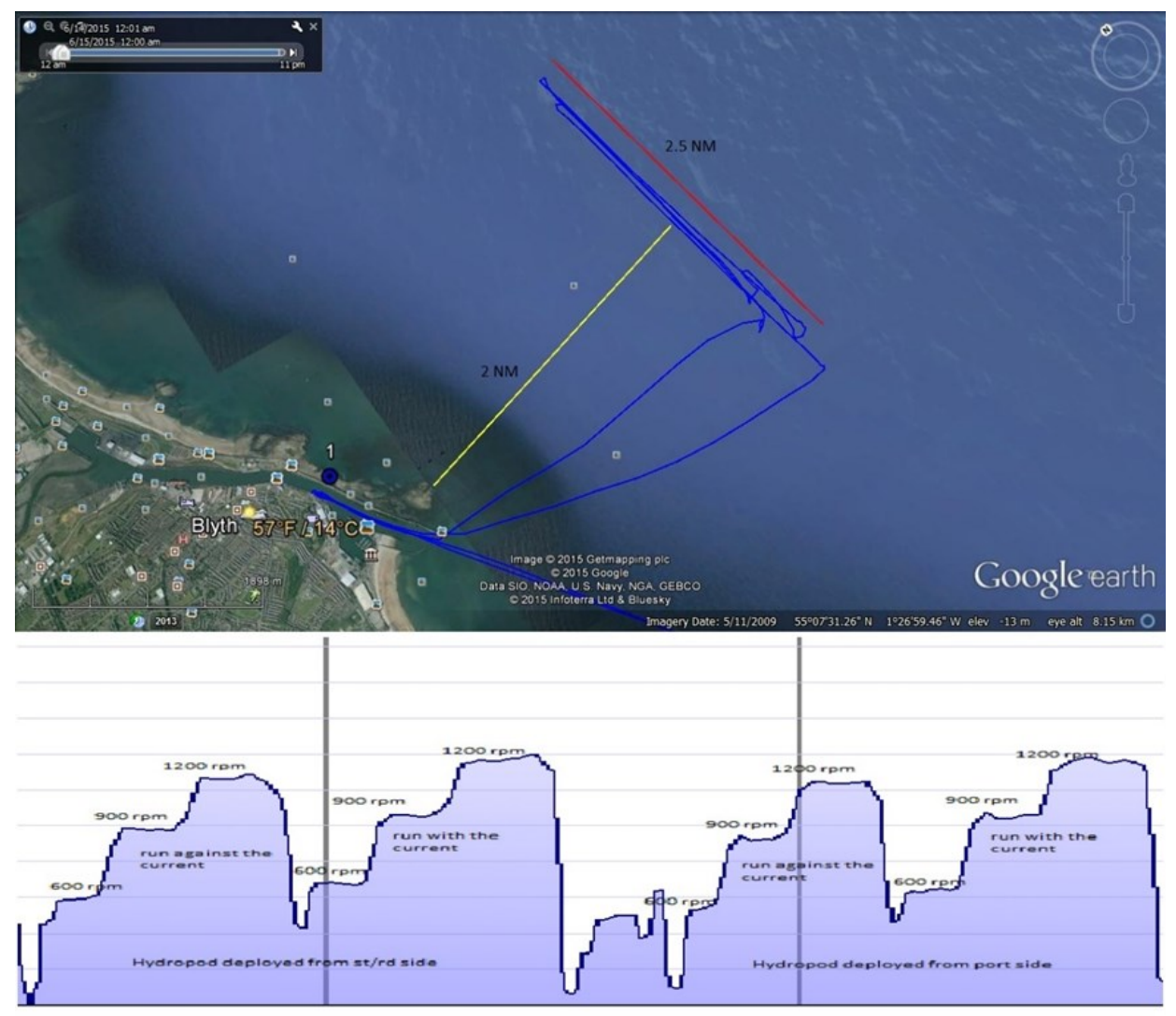

Figure 9 Combination of the runs' trajectory on the map and the gradation in vessel's speed during the first measurements (15-06-2015) using Google Earth.

During the course of the trials shaft torque and speed, vessel speed through water were recorded for calculation of the essential non-dimensional parameters using Equation 1. The detailed fullscale trial test conditions and calculated non-dimensional coefficients are provided in Table 3.

$$
K_{Q}=\frac{Q}{\rho n^{2} D^{5}} \quad \sigma_{n}=\frac{P_{a}+\rho g h_{s}-P_{v}}{0.5 \rho(\pi n D)^{2}}
$$

Equation 1

where, $K_{Q}$ is the propeller torque coefficient, $\rho$ is the density of water, $\mathrm{n}$ is the propeller rotations speed, $Q$ is the torque, $D$ is the diameter of the propeller $\sigma_{s} \sigma_{\mathrm{n}}$ is the cavitation number based on the propeller rotational speed, $P_{A}$ is the Atmospheric pressure, $h_{S} \mathrm{~h}_{\mathrm{s}}$ is the propeller shaft immersion and $P_{v}$ is the vapour pressure. 
Table 3 Full-scale trial test conditions for "The Princess Royal" with old (original) propeller and NPT propeller

\begin{tabular}{|c|c|c|c|c|c|c|c|c|}
\hline DATA / & \multicolumn{8}{|c|}{ VALUES } \\
\hline PARTICULARS & \multicolumn{4}{|c|}{ Old (original) Propeller } & \multicolumn{4}{|c|}{ NPT (replacement) Propeller } \\
\hline $\begin{array}{l}\text { Ship speed through } \\
\text { water, } \mathrm{V}_{\mathrm{S}}(\mathrm{knot})\end{array}$ & 4.5 & 7.2 & 9.5 & 10.9 & 4.7 & 7.5 & 9.9 & 11.1 \\
\hline Engine speed, N (RPM) & 600 & 900 & 1200 & 1500 & 600 & 900 & 1200 & 1500 \\
\hline $\begin{array}{c}\text { Propeller speed, actual, } \\
\mathrm{N}_{\text {Propeller }}(\mathrm{RPM})\end{array}$ & 342.8 & 514.2 & 682.1 & 856.8 & 343.1 & 515.4 & 683.2 & 857.7 \\
\hline Cavitation number, $\sigma_{\mathrm{n}}$ & 1.20 & 0.53 & 0.30 & 0.19 & 1.19 & 0.52 & 0.30 & 0.19 \\
\hline $\begin{array}{c}\text { Torque }(\mathrm{kNm})- \\
\text { (Port side in full scale) }\end{array}$ & 0.3 & 0.6 & 1.0 & 1.6 & 0.3 & 0.6 & 1.0 & 1.7 \\
\hline $\begin{array}{l}\text { Torque Coefficient, } \\
\qquad 10 \mathrm{~K}_{\mathrm{Q}}\end{array}$ & 0.378 & 0.336 & 0.318 & 0.323 & 0.385 & 0.34 & 0.320 & 0.33 \\
\hline
\end{tabular}

\section{Noise measurements}

Hydropod is developed to provide sufficiently accurate noise measurements with an on-board deployed compact system as an alternative to the cumbersome off-board hydrophone array measurements. While it requires further development to be able to replace standard far-field measurements, it presents numerous merits for assessment of underwater noise of a vessel. In order to be able to verify this claim, comparisons are required between off-board measurements and using Hydropod. 
Based on the confidence achieved through the similarity between the Hydropod measurements and off-board hydrophone array measurements, a comparison is made between old propellers and new propellers are presented the evaluate the hydroacoustic performance of NPT applications (Figure 14 to Figure 17). Furthermore, Sound Pressure Levels (SPL) of certain 1/3 octave centre frequencies are extracted to be presented against the ship speed to demonstrate the superiority of the NPT propellers in terms of URN levels given by Figure 18.

Hydropod measurements were made using the Bruel \& Kjaer (B\&K) PULSE Type 3023 data acquisition system with a 6/1 local area network (LAN) interface and the B\&K Type 8103 and 8105 hydrophones. The noise signals were processed by the PULSE lab-shop by using Constant Percentage Bandwidth (CPB) and Fast Fourier Transform (FFT) analyser that were constructed in its dedicated software. The analysed results were presented in $1 / 3$ octave bandwidth for $20 \mathrm{~Hz}$ to $20 \mathrm{kHz}$ and $1 \mathrm{~Hz}$ band levels from $1 \mathrm{~Hz}$ to $6.4 \mathrm{kHz}$.

The noise data acquisition for $1 / 3$ Octave band was conducted by using the waterfall format of the PULSE in order to eliminate the effect of any instantaneous sources. This was achieved by triggering the system every 0.25 seconds for the next measurement. The measurements were recorded for 200 triggers or 50 seconds at $45 \mathrm{kHz}$ sampling rate. 
Detailed background noise measurements were conducted with Hydropod. These measurements not only included the ambient noise measurement but also declutched engine conditions providing further insight into the overall contribution of the engine to the measured levels whilst not coupled to propeller and without any load as given by Figure 13 .

The background noise correction applied depending on the level of the difference following the procedure in [17]Standard. The signal to noise ratio is calculated using Equation 2. Based on the results of the Equation 2 for every $1 / 3^{\text {rd }}$-octave centre frequency, the necessity of applying the background correction is determined. For the measured levels that the difference is smaller than $3 \mathrm{~dB}$, the result is discarded. In the case of a difference between 3 and $10 \mathrm{~dB}$, the results are corrected using Equation 3 and no correction is applied in case of the difference being greater than $10 \mathrm{~dB}$.

$$
\Delta=L_{p+n}-L_{p}
$$

Equation 2

Where; $\Delta$ is the signal plus noise-to-noise ratio, $L_{p+n}$ is the sound pressure level in $\mathrm{dB}$ related to the subject vessel, $L_{p}$ is the background pressure level in $\mathrm{dB}$ uninfluenced by the target vessel.

$$
L_{p}^{\prime}=10 \log \left(10^{\left(\frac{L_{p+n}}{10}\right)}-10^{\left(L_{n} / 10\right)}\right)
$$

Equation 3

where; $L_{p}^{\prime}$ is the background noise corrected sound pressure level (SPL) of the subject vessel and $L_{n}$ is the ambient noise level measurement made on the day of the noise trial. 
The measurements are then normalised to the reference acoustic source level distance. This is achieved by calculation of a total distance of the hydrophone to the acoustic centre of the target vessel using Equation 4. Since Hydropod is fixed onto the vessel, the distance between the hydrophone and propeller is measured during drydock is found to be 1.5 meters and corrected using Equation 5.

$$
d_{\text {Total }}=\sqrt{d_{\text {Horz }}^{2}+d_{\text {Vert }}^{2}}
$$

Equation 4

Where: $d_{\text {Total }}$ is the total normalised distance, $d_{\text {Horz }}$ is the horizontal distance of the hydrophone from the acoustic centre i.e. the hub of the subject propeller and $d_{V e r t}$ is the depth of the hydrophone.

$$
L_{s(r)}=L_{p}^{\prime \prime}+20 \log \left(d_{\text {Total }} / d_{\text {ref }}\right) \quad \text { Equation } 5
$$

Where: $L s(r)$ is the subsurface sound source level at $1 \mathrm{~m}$ reference distance, as a function of run number ( $\mathrm{r})$.

Application of Equation 5 is valid for far field measurements, assuming a spherical propagation from a point source. It is applied for this near field source in this case for two reasons; one is to approximate the noise levels at the reference distance of 1 meter to enable comparisons with the off-board noise measurements and secondly since applied correction with this assumption results in adding $3.52 \mathrm{~dB}$ to the measured levels, it can be offset if found necessary. 
The acquired data is finally converted to equivalent $1 \mathrm{~Hz}$ bandwidth $S P L$ in each $1 / 3$ Octave band using the formula recommended by [25]as in Equation 6.

$$
S P L_{1}=S P L_{m}-10 \log \Delta f \quad \text { Equation } 6
$$

During post-processing, the acoustic data derived from the port and starboard aspect measurements were averaged for north and south runs. Thus, for each RPM, four individual runs are conducted and eventually averaged for the calculation of the ultimate noise levels presented throughout the paper. This was carried out after the transformation from $1 / 3$ octave band to an equivalent $1 \mathrm{~Hz}$ bandwidth sound source level in $\mathrm{dB}$ relative to $1 \mu P a(d B$ re $1 \mu P a$ at $1 \mathrm{~m})$ from $20 \mathrm{~Hz}$ to $20 \mathrm{kHz}$.

One unfortunate technical difficulty occurred during the tests conducted with Hydropod was the malfunction of B\&K 8105 Hydrophone following the trials with the old propeller. This was rectified by manufacturing a new tip section that enabled the attachment of $\mathrm{B} \& \mathrm{~K} 8103$ hydrophone to the Hydropod. The only consequence of this was the change in the hydrophone self-noise properties due to the B\&K 8105 being a larger device compared to the B\&K 8103 .

Whilst self-noise associated with hydrophones may have components such as the "electronic noise" and "hydrodynamic flow noise". In the context of this case, dominating factor effecting the measurements has been the hydrodynamic flow noise over the hydrophone as it was not enclosed within a dome or casing. The effect of hydrodynamic flow noise can be rectified by conducting hydro acoustic tests at a cavitation tunnel. This would be in line with the recently defined procedures for model testing, where facility transfer functions are used for correcting direct noise measurements. 


\subsection{Evaluation of the Hydropod concept}

First set of trials were conducted with the old propellers of "The Princess Royal", for which ample noise trial data was readily available. The trials were conducted according to ISO, (2016) by $[23,26]$ (referred as Off-board). The comparisons are presented from Figure 10 to Figure 12.

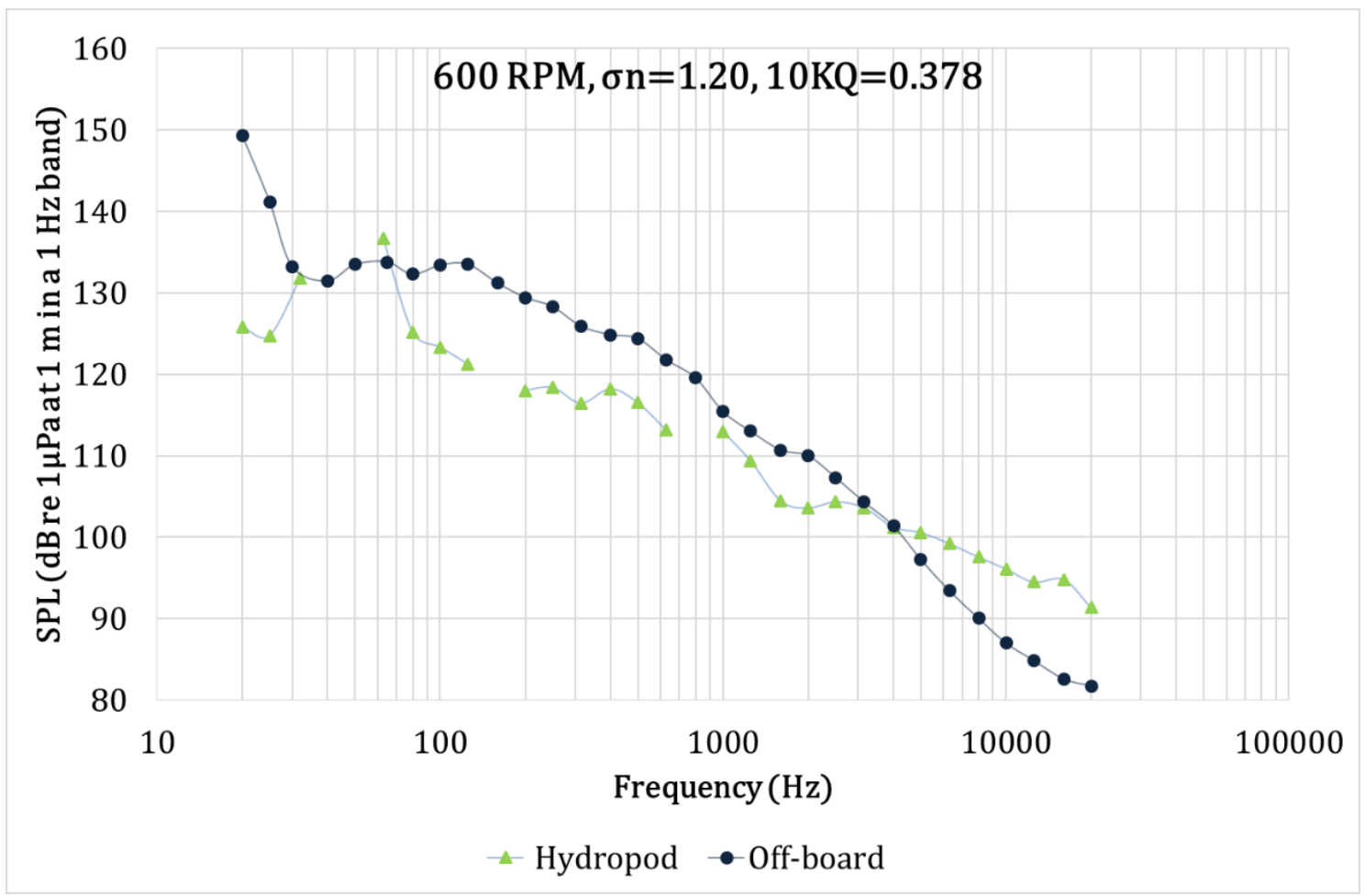

Figure 10 Comparison of Hydropod and off-board analysed net noise data at 600 engine rpm. 


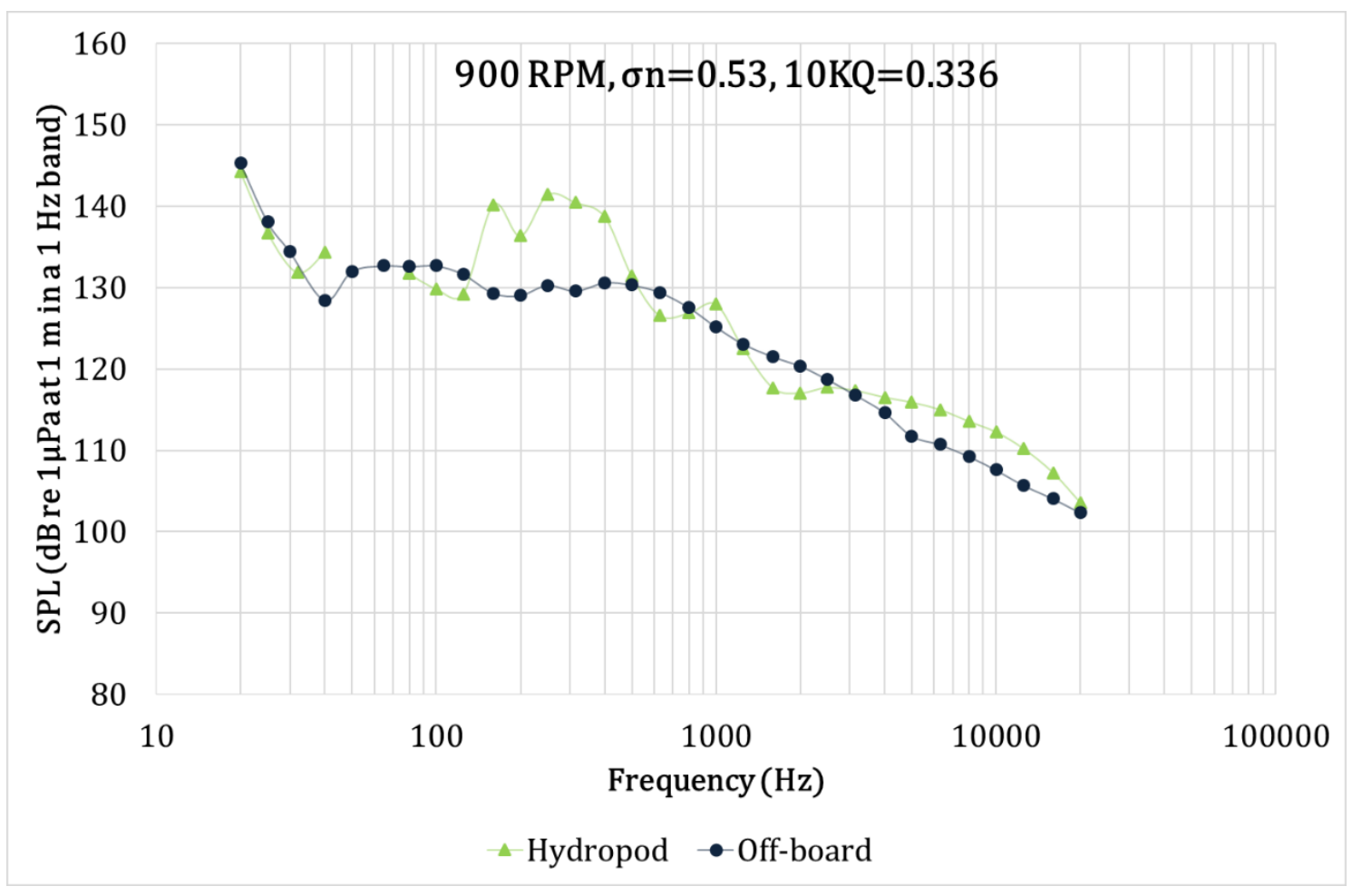

Figure 11 Comparison of Hydropod and off-board analysed net noise data at 900 engine rpm.

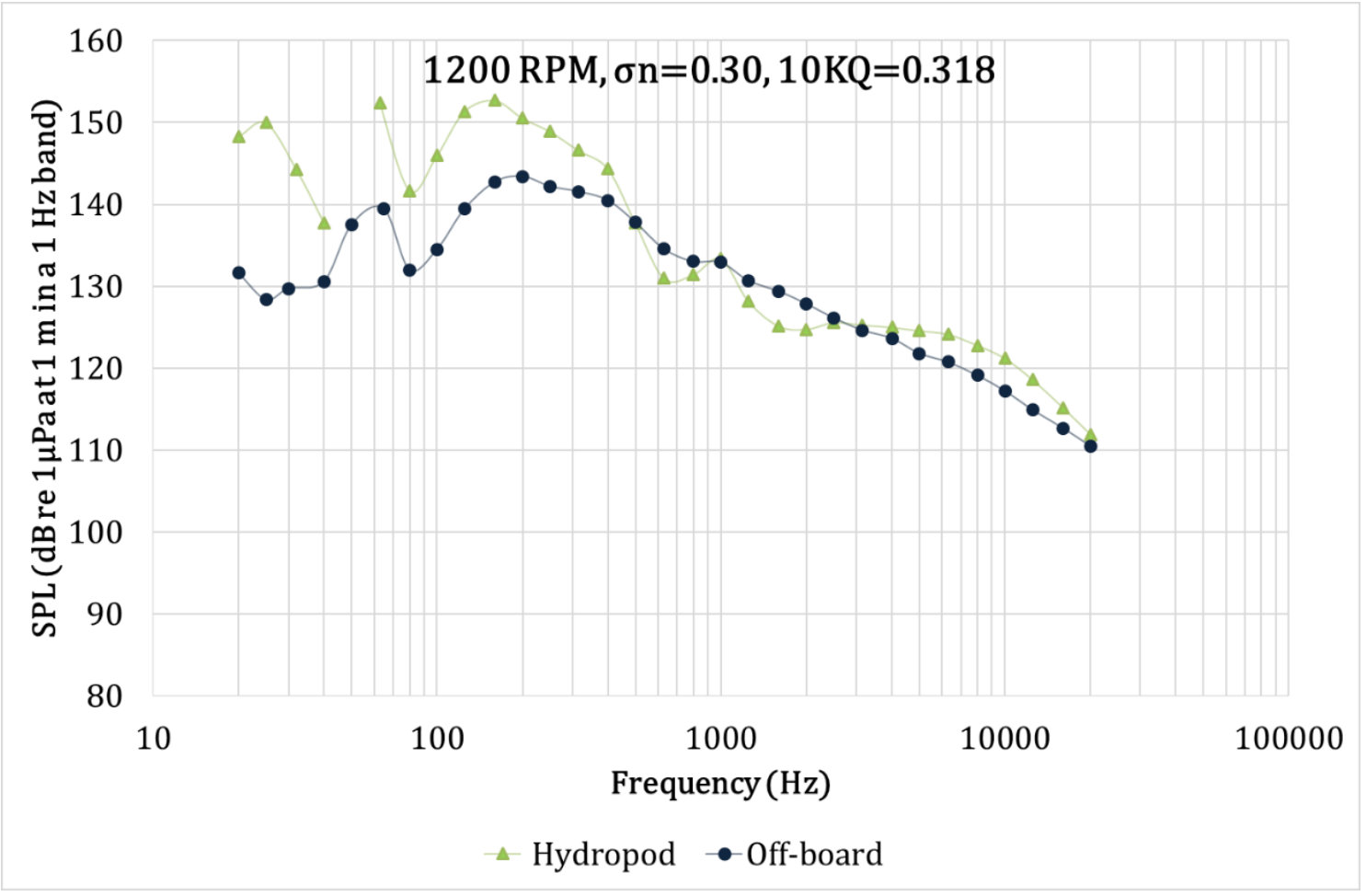


Figure 12 Comparison of Hydropod and off-board analysed net noise data at 1200 engine rpm.

The comparisons of the sound pressure levels presented through Figure 10 to Figure 12 indicate that the general trend and magnitude of the noise measurements made with the Hydropod show significant similarities to those of the measurements made with the conventional off-board hydrophone array system. Some disagreements can be observed due to the characterization of the dominating acoustic sources and hydrodynamic flow noise of the Hydropod system. Within this framework, as shown in Figure 10, which presents the comparative noise data for noncavitating condition, there is a different trend between the noise level data obtained from the Hydropod and off-board measurements. This may be attributed to the dominating acoustical sources. Namely, the condition was dominated by the dipole sources induced by vortex shedding, fluctuating loads, turbulent trailing edge noise, flow induced vibrations and quadrupole type sources induced by turbulent fluid motions as there was no cavitation present on the propeller. Therefore the near-field measurement data of the Hydropod were dominated by these types of source, due to the inherent acoustical analogy whilst the far field off-board measurement was much less affected by these sources due to their low radiation efficiency with distance [27]. These acoustical principles are of course interrelated with machinery noise which is the dominant noise source in non-cavitating condition.

Figure 11 and Figure 12 shows the comparative noise data for the cavitating conditions, which display good agreement between the two type measurements except the hump on the hydropod measurements in the mid-frequency region. This can be atributed to the hydrodynamic flow noise of the hydrophone [28]. The hump moves towards the higher spectral levels and lower frequency region with the increasing speed of the vessel following the theory proposed by Haddle \& Skudryzyk based on the experimental study carried out with different size and diameter hydrophones that were tested at different speeds. The comparisons made for the 900RPM show only disagreement from 125 to $500 \mathrm{~Hz}$ because of the hydrodynamic flow noise 
hump of the Hydropod. On the other hand, for 1200RPM case, the comparisons are in disagreement both because of the hydrodynamic flow noise hump as well as well-known difficulties with off-board measurements particularly in low frequency region $(1-100 \mathrm{~Hz})$ [13].

During the trials, the ambient noise level measurements were conducted for the background noise correction. These included additional noise measurements with the declutched engine and results are shown in Figure 13. In the declutched condition although there was no load on the engine, the measurements provide invaluable insight into the contribution of the engine (without load) to the noise spectra. In addition to that, as shown in Figure 13, the comparison of the background levels at 600 RPM and 900 RPM presents significant elevation of the spectral level for 900RPM with the inception of the cavitation. The only remark that has been left unanswered after the execution of the background levels was the peak at $50 \mathrm{~Hz}$ in the background noise measurements, which resulted in the discontinuity of the measured data after the background noise correction. The detailed investigation carried out leaves the electric generator as the only suspect, but this cannot be confirmed, as it required measurement without the electrical source, which was not possible during the test schedule 


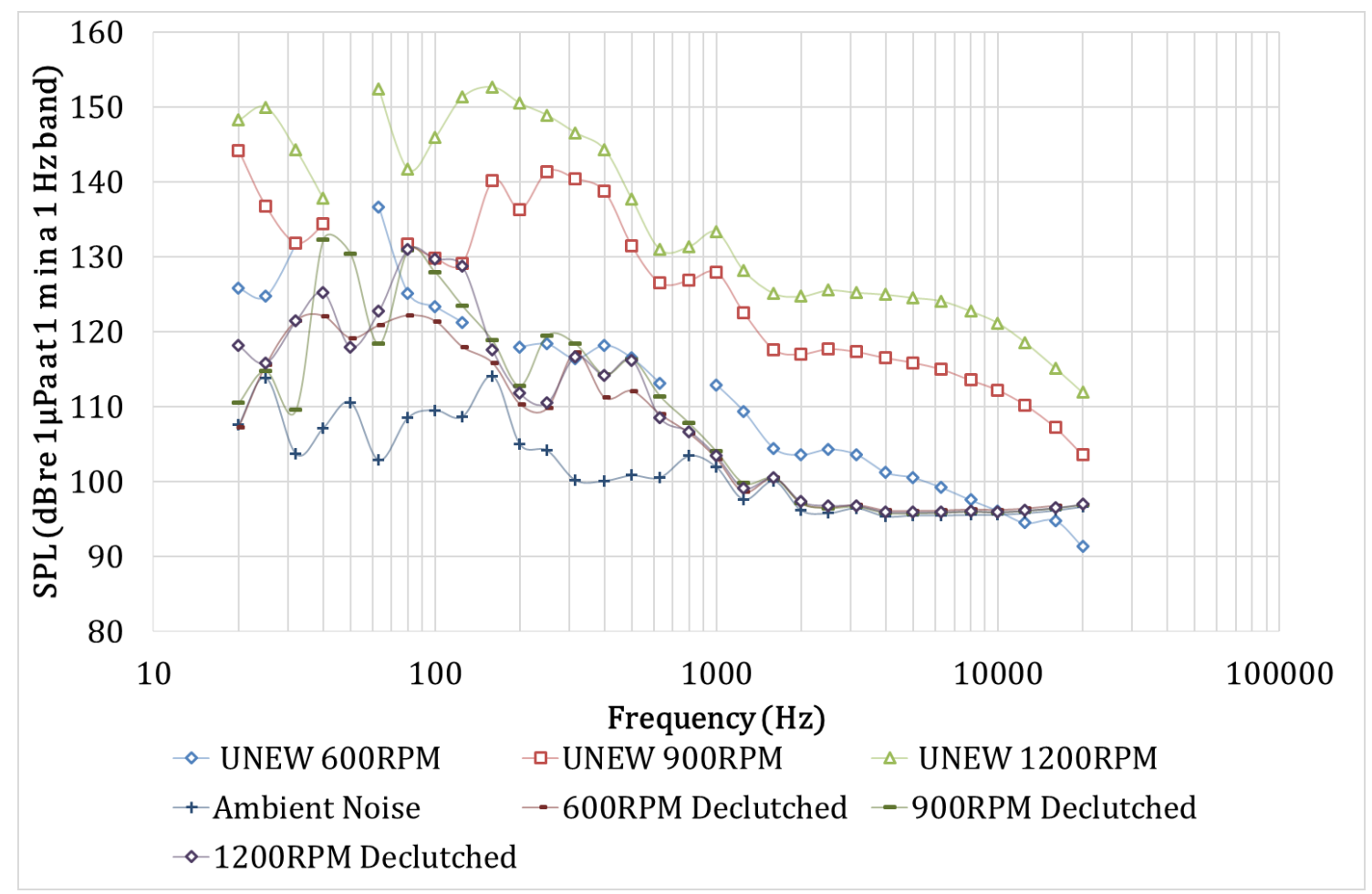

Figure 13 Ambient and declutched engine rpm noise levels together with net noise level measurements while the vessel is operating.

\subsection{Assessment of the New Profile Technology (NPT) propellers}

Following the execution of the trials with the old propellers using Hydropod, the vessel was dry-docked in June 2015 and her propellers were replaced with the New Profile Technology (NPT) propellers. The trials were then repeated with the new set of propellers. This enabled the evaluation of the performance of the old and new propellers in terms of their hydro-acoustic performance.

The full-scale trials were repeated at identical conditions except the following differences. The first difference was the hull condition. During the first trials with the old propellers, the hull was fouled while it was immaculately clean during the second trials with the new propellers as the vessel was just dry-docked. Whilst the difference of the fouling condition of the hull is a source of uncertainty between measurements, fouling may be considered to be a secondary 
cause regarding the noise radiated by a cavitating propeller. The second difference was the unfortunate malfunctioning of the B\&K 8105 hydrophone during the pre-test calibration. As stated earlier, this issue was rectified by swapping the B\&K 8105 with B\&K 8103 in the hydropod. As a drawback of this substitution, the hydrodynamic flow noise of the hydrophones were different showing humps at different frequencies due to their difference size, which reflected on the results presented in Figure 14 to Figure 17 comparing the noise levels measured by the Hydropod with the two different sets of propellers (i.e. original old propeller and new NPT propeller). The frequency response of the hydrophones were corrected by the certified calibration data. This procedure was applied for both hydrophones and thus has no significant effect over the measurements.

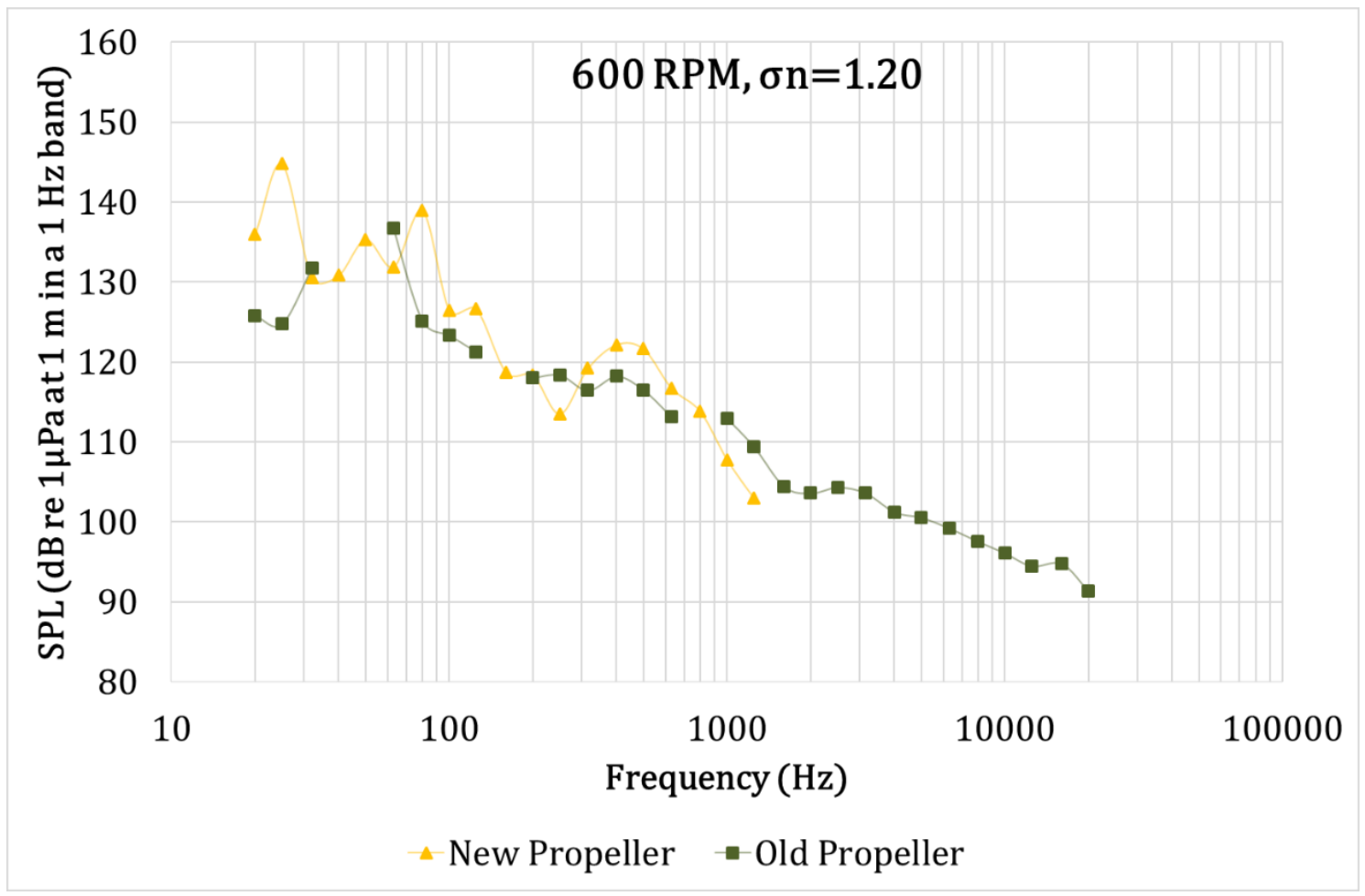

Figure 14 Comparison between old and new propellers at 600 engine rpm, using the Hydropod. 


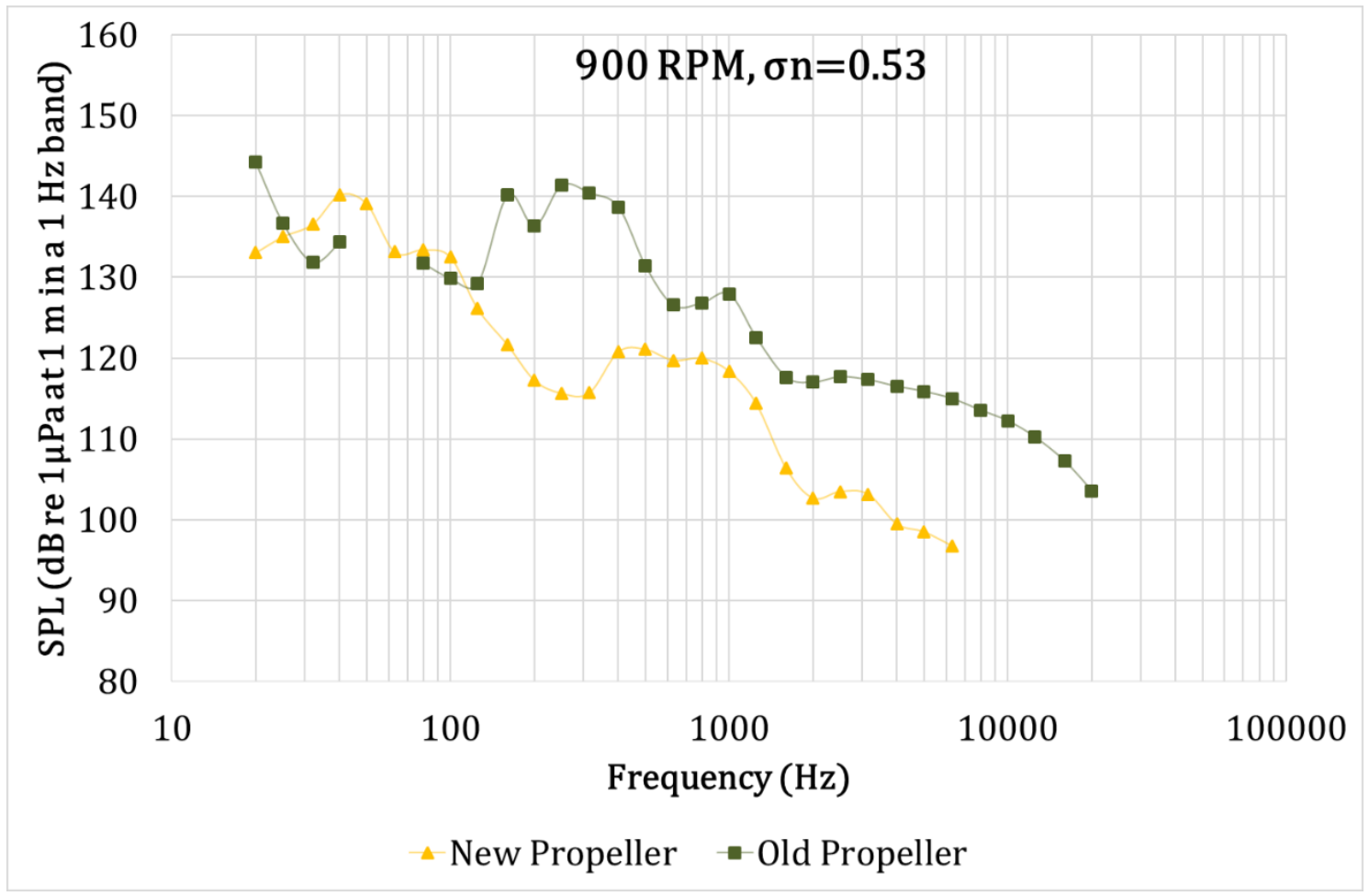

Figure 15 Comparison between old and new propellers at 900 engine rpm, using the Hydropod.

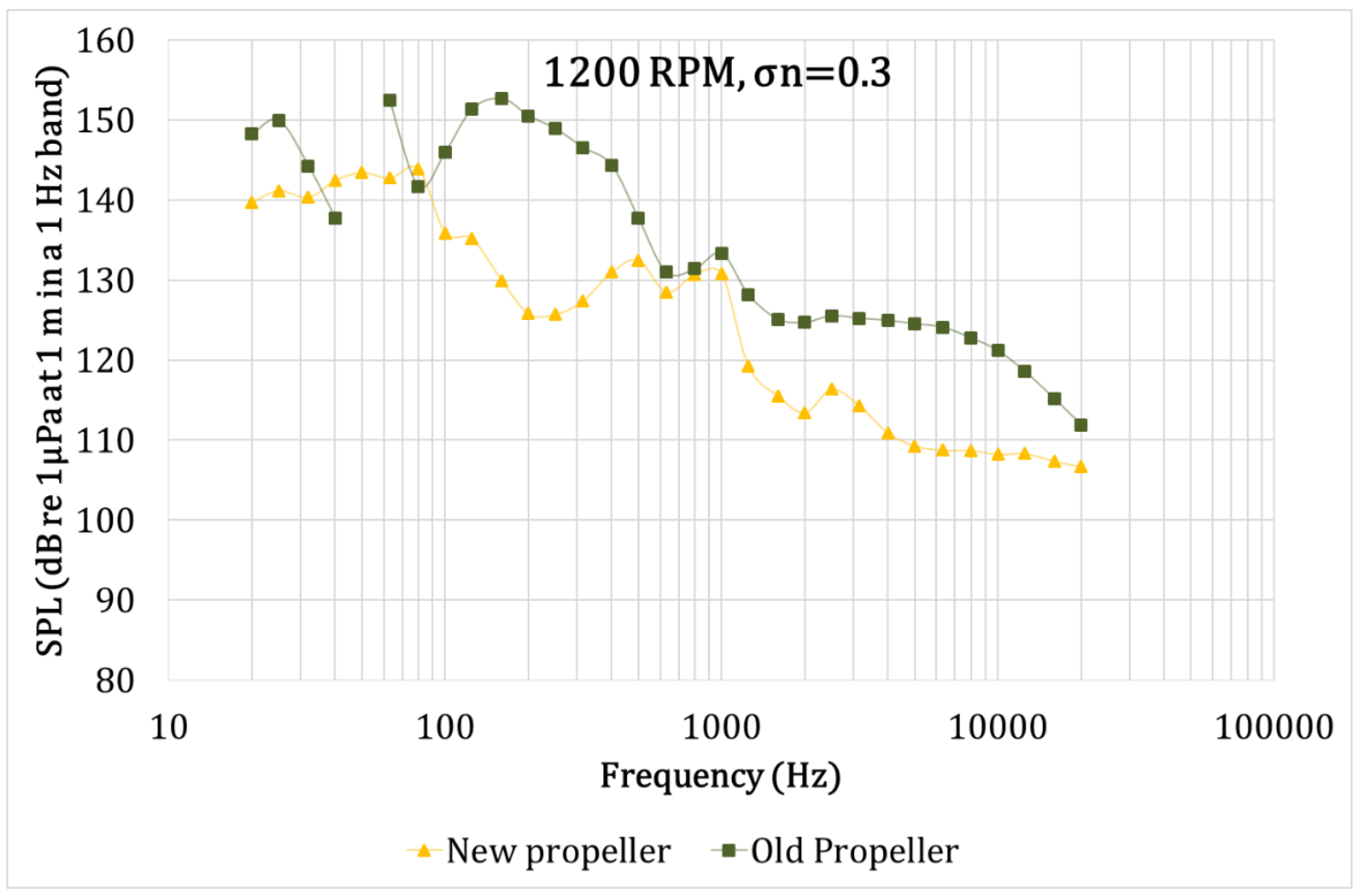

Figure 16 Comparison between old and new propellers at 1200 engine rpm, using the Hydropod 


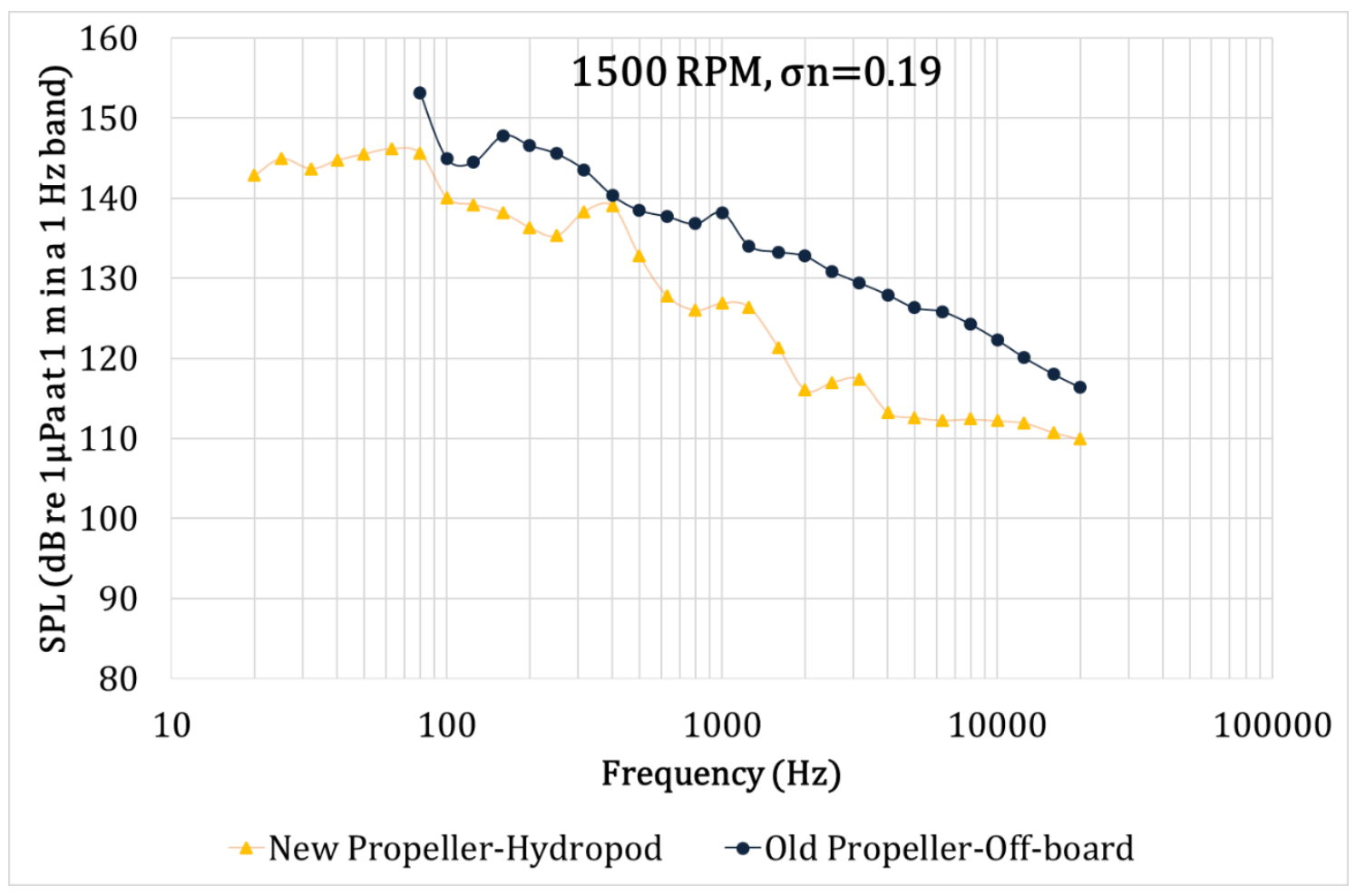

Figure 17 Comparison between old and new propellers at 1500 engine rpm, using two different techniques (New propeller; Hydropod, Old propeller; SOTON data)

The comparison of the measured data for the non-cavitating condition of the old and new propellers at 600 RPM present similar trends in terms of their levels and overall characteristics as shown in Figure 14. On the other hand, when the cavitation was incepted and developed further, significant divergence of the noise spectra of the two sets of propellers can be observed. As shown in Figure 15 and 16 for 900 RPM and 1200 RPM, respectively, the noise levels of the new NPT propeller are significantly lower than those of the old propeller with a clear precedence. The reduction is observed to be at an average of $10 \mathrm{~dB}$ indicating the great potential of the NPT propeller for better noise performance.

Figure 17 shows further comparisons of the noise data for the two propeller at 1500RPM. For this condition, since the Hydropod measurements was not conducted with the old propeller, the comparison was made by using the off-board hydrophone array measurement data with the old propeller. However, as shown in Figure 17, this comparison also supports the findings for the 
900 and 1200 RPM conditions by displaying significant noise reduction with the NPT propeller through the whole frequency range.

In order to compare the noise levels of the two propellers as a function of the ship speeds Figure 18 is included. As shown in this figure the extracted SPL values at certain $1 / 3^{\text {rd }}$-octave centre frequencies against the ship speed is further confirming the significant improvement achieved in terms of hydro-acoustic performance with the new set of propellers.
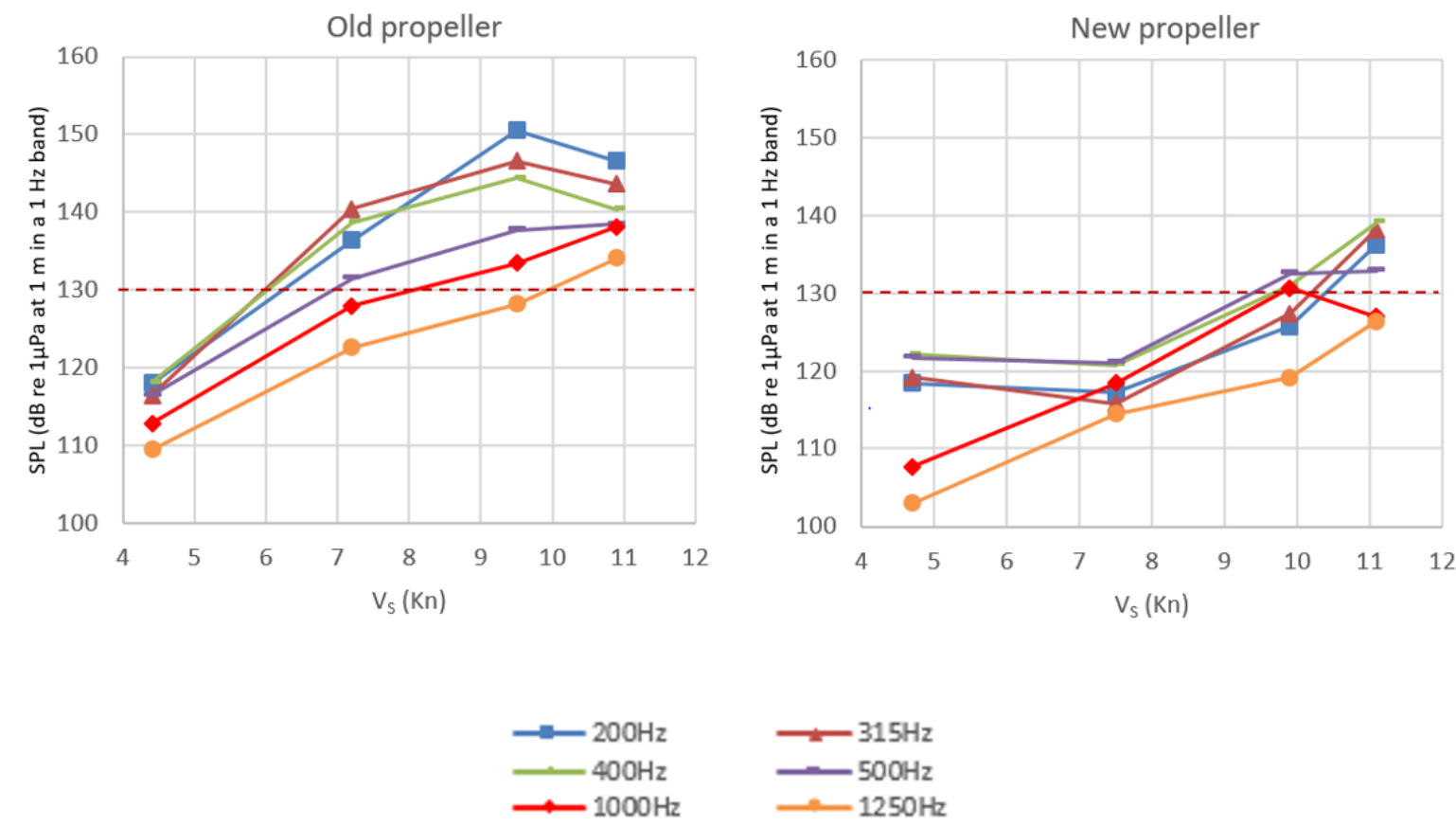

Figure 18 Comparative sound pressure levels measured at different ship speeds

The above presented results obtained using the experimental Hydropod device are proven to be consistent and thus, the method can be claimed to be reliable for future noise and cavitation recordings and investigations on board merchant and navy vessels. The method is practical, flexible and cheaper compared to classic three-hydrophone array system and provides similar acoustic values coupled with relevant cavitation observations. Hence, classification societies 
may review and study this technique to utilise for upcoming amendments to existing standards. Furthermore, it is a self-contained method without requiring any auxiliary vessels or noise range facilities because all recording and analysing equipment is accommodated within the subject vessel.

As far as the noise characteristics are concerned, overall it can be concluded that the NPT propellers appear to be much quieter compared to the old propeller. At non-cavitating speeds, this difference is not significant, but when cavitation is incepted and developed further, clear divergent results are obtained. Moreover, ambient and engine noise contribution appear to have a major role in the overall underwater sound emission, but propeller noise at cavitating speeds dominates the entire frequency spectrum.

\section{Cavitation observations}

The underwater camera located on the Hydropod also provided accompanying qualitative information on the cavitation experienced by the propeller throughout the measurements. The cavitation observations were most useful to interpret the measured noise levels and understand the reasons behind the differences in the measured levels.

The underwater camera utilised was a mass-market brand for extreme sports. Since subject research vessel operated with a high-speed diesel engine, the camera was configured to operate at the highest frame rate by minimising the quality. Although shutter speed imposed a limit for better resolution, the observations were still highly informative and insightful. The recordings were also profoundly influenced by the natural light availability and water clarity. As can be seen from Figure 19 to Figure 21, which show the comparative starboard views of the old and 
new propellers for 600, 900 and 1200 RPM, the water conditions during the second trials were not as clear as for the first trails with relatively blurry images. The recorded cavitation observations are also converted to slow motion videos and provided in Appendix A.

Despite the blurry videos during the second trial, the comparison of the cavitation performance for the two sets of propellers clearly demonstrated improvements achieved with new NPT (New Profile Technology) propellers. The progression of tip-vortex cavitation was observed to be significantly reduced as shown in Figure 19 to Figure 21.

Figure 19, in this respect, presents the 600 RPM condition for both propellers. Although the old propeller showed intermittent and inconsistent inception of cavitation during this condition, there was no trace of cavitation for the NPT propellers. However, the 900 RPM condition shown in Figure 20 displayed significant difference in terms of the tip vortex cavitation diameter for the two sets of propellers. Whilst the old propeller experienced a well-developed tip vortex cavitation travelling in the slipstream all the way to the rudder and probably after the stern, the NPT propellers only experienced intermittent tip vortex cavitation in the wake shadow area. For the 1200 RPM condition similar findings to the 900 RPM case was observed with increased strength and diameter of tip vortex cavitation for the old propellers and relatively stronger tip vortex cavitation with weak slipstream traces for the NPT propellers as shown in Figure 21.

Following the improvements implemented during the dry docking period, successful cavitation observations were also made for 1500 RPM condition during the second trials. The slow motion videos of this condition is also provided in Appendix A. 
The observed difference between two propeller sets in terms of cavitation presence is closely interrelated to the measured spectral levels. For the non-cavitating 600RPM condition, the measured spectral levels are as the dominating noise sources such as the propeller blade thickness and engine noise are similar for both propellers. This is reflected in the measured similar spectral levels as can be seen in Figure 14. However, with the inception of cavitation, experienced cavitation extent and dynamics start to differ for the two sets of propellers. This consequently impacts the measured noise levels. For 900RPM condition, tip vortex cavitation is the predominantly observed for both propellers with different strength and diameter. The tip vortex cavitation for the old propeller is rather bigger in diameter and continuous whereas the one experienced by the new propeller has a smaller diameter and intermittent in the wake shadow area. The significant difference in terms of the tip vortex cavitation strength is also captured by the noise measurements as can be seen in Figure 15 by the elevated levels experienced by the old propeller. For 1200RPM condition, the old propeller experiences relatively strong suction side "Sheet Cavitation" emanating from the entire blade leading edge with increased extent (hub to tip) terminated at the blade tip by rolling-up in the form of "Trailing Tip Vortex" extending to the rudder. Partial "break-up of the sheet cavitation" as well as occasional appearance of "Hub Vortex Cavitation" and "Hull-Propeller Vortex" cavitation were observed. The new propeller for this condition shows mainly vortex type cavitation. Welldeveloped tip vortex with a thickness of $5-10 \mathrm{~mm}$ extends continuously in the downstream while at the leading edge of the blade. The difference in terms of the extent of the cavitation and dynamics also reveals itself in the measured acoustic spectra in Figure 16. 


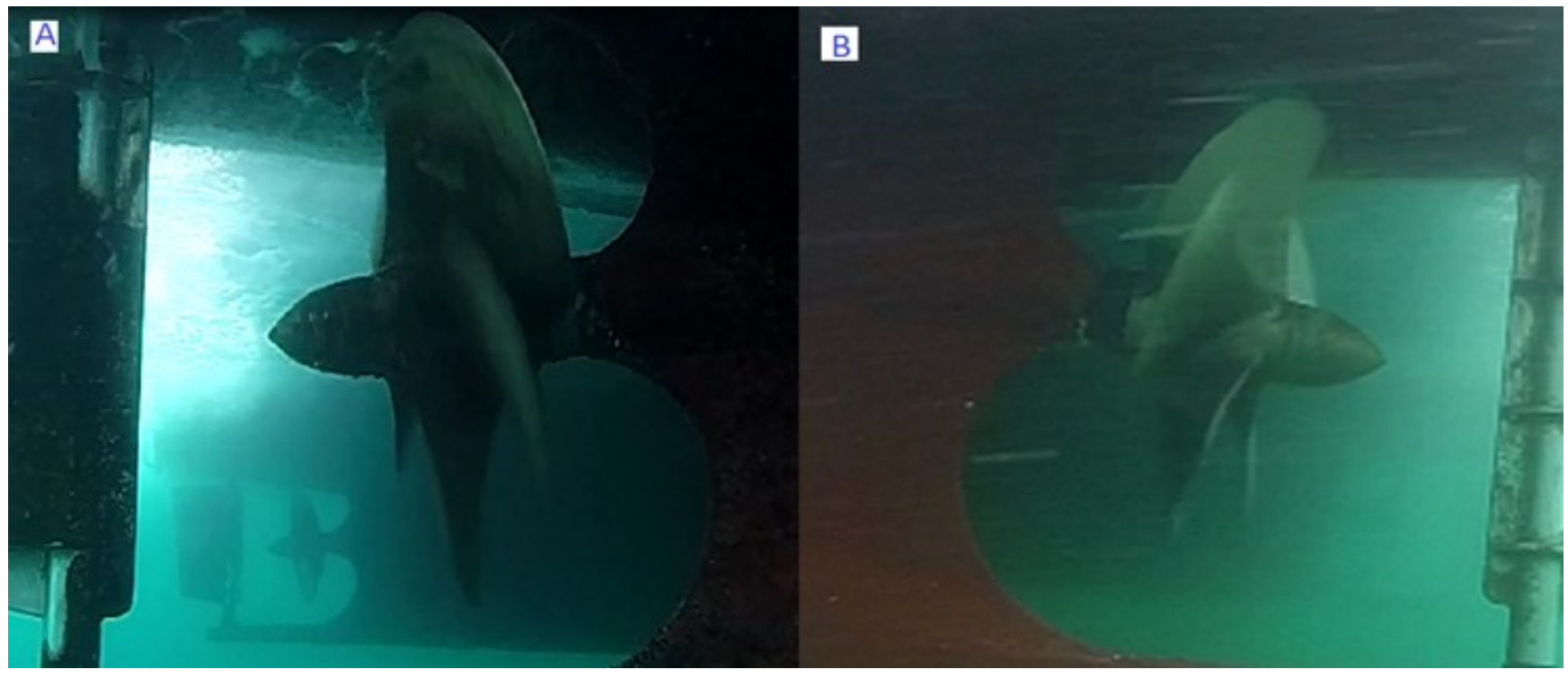

Figure 19 Cavitation observation snapshots of old $[\mathrm{A}]$ and new $[\mathrm{B}]$ propellers at 600 engine rpm.

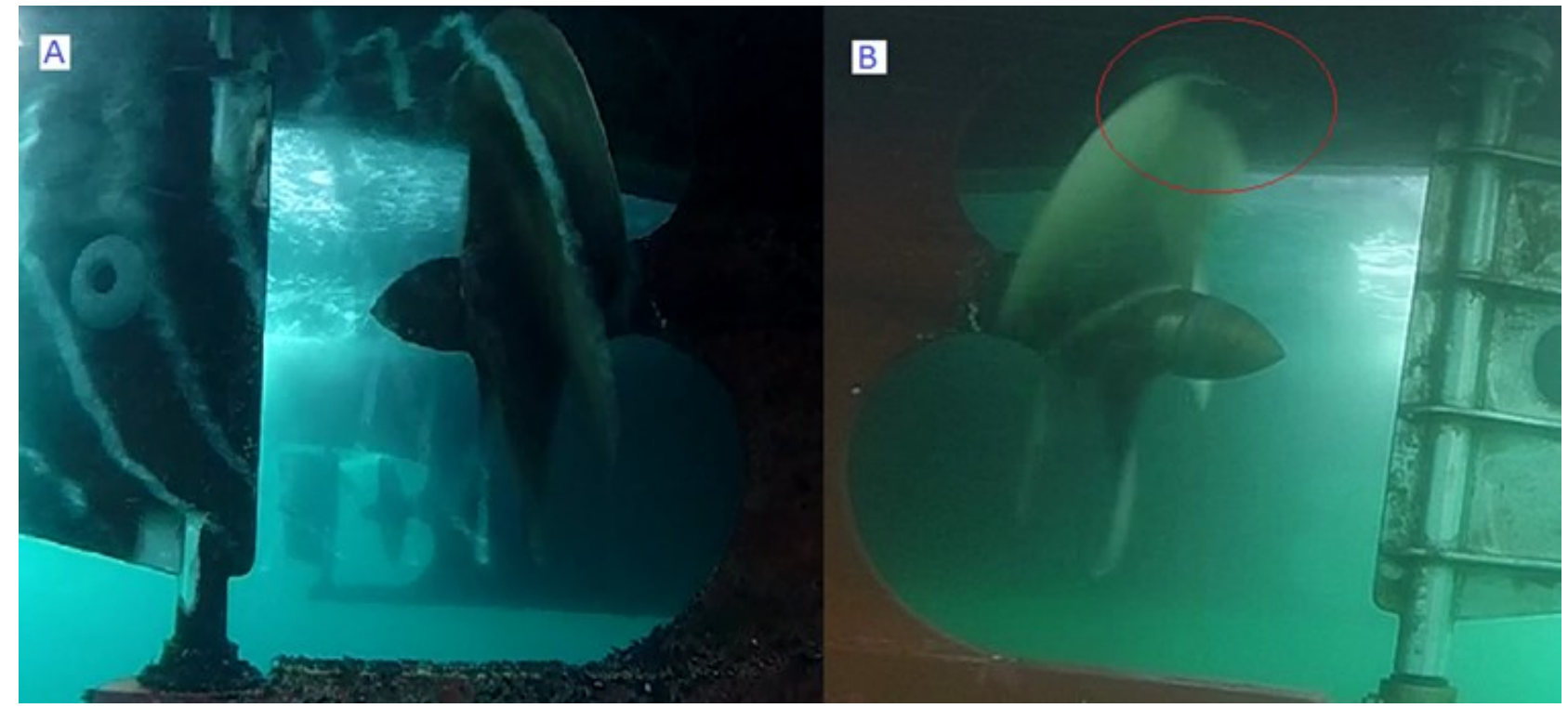

Figure 20 Cavitation observation snapshots of old $[A]$ and new $[B]$ propellers at 900 engine rpm. 


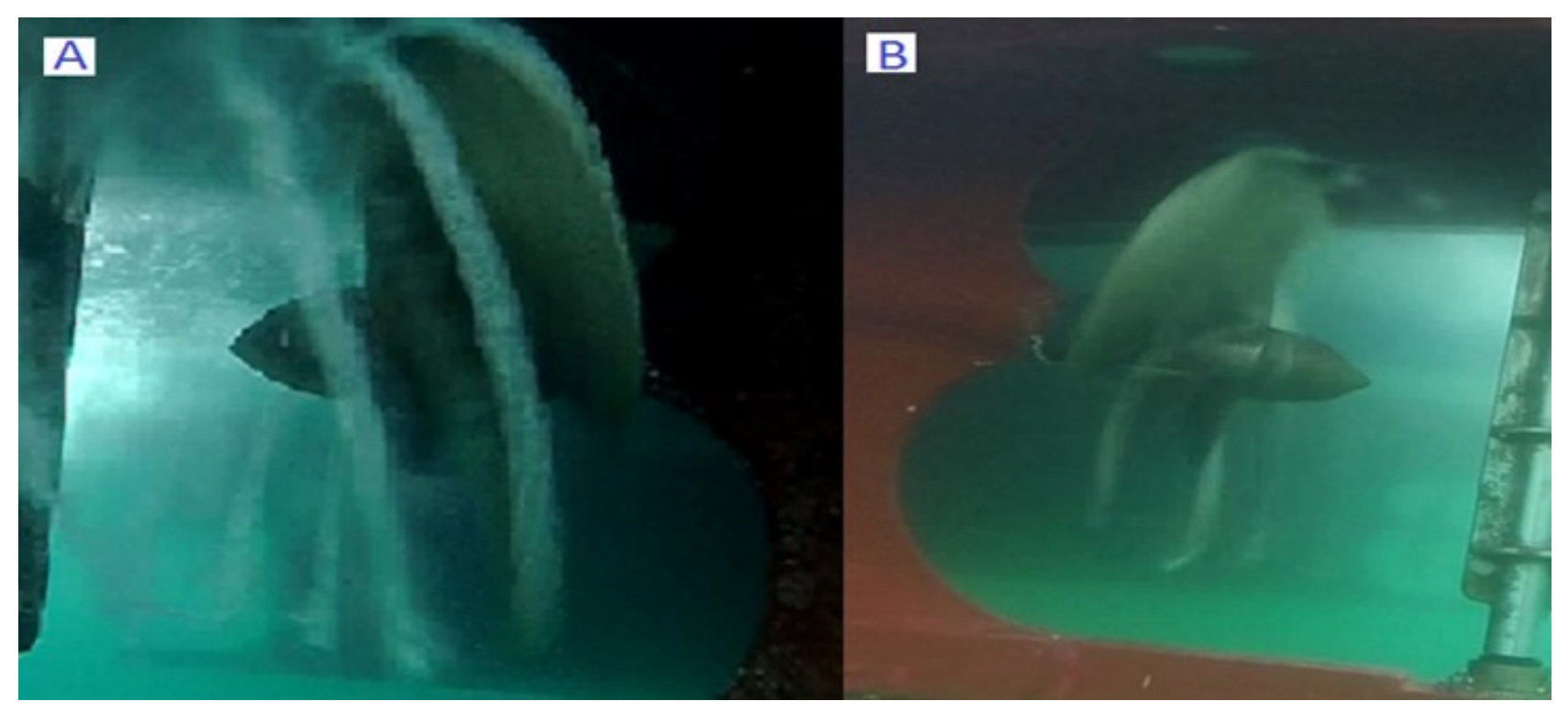

Figure 21 Cavitation observation snapshots of old $[A]$ and new $[B]$ propellers at 1200 engine rpm.

Overall, the cavitation observations made with the Hydropod supported the URN measurements showing significant difference in terms of the cavitation presence. Especially in terms of tip-vortex cavitation inception, development and slipstream extension, it was apparent that the new propellers of the vessel presented a superior performance compared to the old propellers.

\section{Conclusion}

The rising environmental awareness regarding the anthropological impact on marine fauna may result in potential limits to be introduced regarding the noise levels of commercial vessels. The assessment procedure by conducting off-board hydrophone arrays is cumbersome and costly. To address this issue, this paper presented a plausible noise assessment method by using an onboard deployable device that consists of a hydrophone and an underwater camera. This practical on-board device, named as Hydropod, has been proposed to measure the URN of a 
ship propeller and observe its cavitation as well as to demonstrate the functionality of the Hydropod on board a research catamaran. The device was used to assess the underwater noise and cavitation assessment of two different types of propeller on the research vessel. Based on the study presented it can be concluded that:

- Hydropod based on-board technique can be a practical and attractive alternative to classical off-board noise measurement technique for ball-park noise and cavitation assessment

- Hydropod device was able to measure the underwater noise spectra of the research vessel with comparable sound pressure level and frequency distribution of the noise spectra obtained by the off-board measurement.

- The device was able to distinguish clearly the different noise and cavitation characteristics of the two different types of propeller used on the research vessel in favour of the new NPT based propellers.

- Hydropod can be an attractive cavitation observation device depending on lighting and seawater quality conditions.

- Hydropod device can be further developed to apply other commercial vessels and its self-induced noise can be filtered based on further investigations.

\section{Acknowledgments}

The Authors gratefully acknowledge the help and assistance received from the Emerson Cavitation Tunnel Team and "The Princess Royal" staff during the trials. 


\section{Reference}

[1] J. a. Hildebrand, Anthropogenic and natural sources of ambient noise in the ocean, Mar. Ecol. Prog. Ser. 395 (2009) 5-20. doi:10.3354/meps08353.

[2] G. V. Frisk, Noiseonomics: The relationship between ambient noise levels in the sea and global economic trends, Sci. Rep. 2 (2012) 2-5. doi:10.1038/srep00437.

[3] G.M. Wenz, Acoustic Ambient Noise in the Ocean: Spectra and Sources, J. Acoust. Soc. Am. 34 (1962) 1936-1956. doi:10.1121/1.1909155.

[4] N.R. Chapman, A. Price, Low frequency deep ocean ambient noise trend in the Northeast Pacific Ocean, J. Acoust. Soc. Am. 129 (2011) EL161-EL165. doi:10.1121/1.3567084.

[5] M.A. McDonald, J.A. Hildebrand, S.M. Wiggins, D. Ross, A 50 year comparison of ambient ocean noise near San Clemente Island: a bathymetrically complex coastal region off Southern California., J. Acoust. Soc. Am. 124 (2008) 1985-1992. doi:10.1121/1.2967889.

[6] J.A. Hildebrand, Impacts of Anthropogenic Sound, in: Mar. Mammal Res. Conserv. beyond Cris., The Johns Hopkins University Press, Baltimore, Maryland, 2005: pp. 101124. doi:10.1017/CBO9781107415324.004.

[7] M. Simmonds, S. Dolman, L. Weilgart, Oceans of noise: a WDCS Science Report, in: Whale Dolphin Conserv. Soc., 1997: p. 168. doi:10.1109/OCEANS.1997.624092.

[8] P. White, F. Pace, The Impact of Underwater Ship Noise on Marine Mammals, 1st IMarEST Sh. Noise Vib. Conf. (2010).

[9] W.J.W. Richardson, C.R. Greene, Jr., C.I.C. Malme, D.H.D. Thomson, C.G. Jr, C.I.C. Malme, D.H.D. Thomson, Marine mammals and noise, Elsevier Science, 2013. https://books.google.co.uk/books?hl=en\&lr=\&id=j6bYBAAAQBAJ\&oi=fnd\&pg=PP1 
$\& d q=$ Marine + mammals + and + noise $\&$ ots $=$ B9RtHnguUd\&sig $=$ Kj3sGq6GB6Sas4dgD8s 67W7pmdU (accessed January 27, 2016).

[10] IMO, Noise From Commerical Shipping and Its Adverse Impact on Marine LifeDevelopment of an international standard for measurement of underwater noise radiated from merchant ships, MARINE ENVIRONMENT PROTECTION COMMITTEE (MEPC) 62nd session Agenda item 19, Submitted by the International Organization for Standardization (ISO), MEPC 62/19, 2011

[11] IMO, Noise from commercial shipping and its adverse impacts on marine life, Marine Environment Protection Committee, International Maritime Organization (MEPC), 66th Session, Agenda item 17, 2013.

[12] A.J. Van der Graaf, M.A. Ainslie, M. André, K. Brensing, J. Dalen, R.P.A. Dekeling, S. Robinson, M.L. Tasker, F. Thomsen, S. Werner, European Marine Strategy Framework Directive- Good Environmental Status (MSFD GES), 2012.

[13] A.M. Rodríguez, R. Salinas Mullor, P. Beltrán Palomo, E. Baudin, V. Lamaison, New European Underwater Noise Measurement Standard Developed in the AQUO Project, MTS/IEEE Ocean. Conf. (2015) 1-6.

[14] DNV, SILENT Class Notation, Rules Classif. Ships. (2010) 1-18. doi:10.1038/147264c0.

[15] R.B. Mitson, Underwater noise of research vessels: Review and recommendation, Coop. Res. Report, ACOUSTEC. 209: (1995) $65 . \quad$ http://www.brasilrounds.gov.br/round9/round9/guias_R9/sismica_R9/Bibliografia/Mitson 1995 Underwater noise of research vessels.pdf (accessed May 2, 2014).

[16] ANSI, Quantities and Procedures for Description and Measurement of Underwater Sound from Ships - Part 1 :General Requirements, Acoustical Society of America, Part 1: General Requirements, 2009. doi:10.1016/0003-6870(91)90314-8. 
[17] ISO, Acoustics — Quantities and Procedures for Description and Measurement of Underwater Sound From Ships, in: Part 1 Gen. Requir. Meas. Deep Water, BSI Stand. Publ. PD ISO/PAS 17208-12016, 2016.

[18] M. Atlar, B. Aktas, R. Sampson, K.-C. Seo, I.M. Viola, P. Fitzsimmons, C. Fetherstonhaugh, A Multi-Porpose Marine Science \& Technology Research Vessel for Full-Scale Observations and Measurements, in: Adv. Meas. Technol. AMT'13, 2013.

[19] N. Sasaki, G. Patience, Evolution of high efficiency propeller with new blade section, in: Mot. Sh. Conf., Bilbao, Spain, 2005.

[20] B. Aktas, A Systematic Experimental Approach to Cavitation Noise Prediction of Marine Propellers, Newcastle University, PhD Thesis, 2016.

[21] NATO, Standards For Use When Measuring And Reporting Radiated Noise Characteristics Of Surface Ships, Submarines, Helicopters, Etc. In Relation to SONAR Detection and Torpedo Acqusisition Risk, North Atl. Treaty Organ. STANAG 113 (1995).

[22] ITTC, Full Scale Measurements Speed and Power Trials Preparation and Conduct of Speed/Power Trials, Recommended Procedures and Guidelines, 7.5-04-01-01.1, 2005.

[23] A. Brooker, V. Humphrey, Measurement of Radiated Underwater Noise from a Small Research Vessel in Shallow Water, in: A. Yücel Odabaşı Colloq. Ser. 1 St Int. Meet. Propeller Noise Vib., Istanbul, $\quad$ Turkey, 2014. http://www.ayocol.itu.edu.tr/files/AYOCOL_2014_Proceedings.pdf\#page=57 (accessed February 23, 2015).

[24] B. Aktas, M. Atlar, S. Turkmen, W. Shi, R. Sampson, E. Korkut, P. Fitzsimmons, Propeller cavitation noise investigations of a research vessel using medium size cavitation tunnel tests and full-scale trials, Ocean Eng. 120 (2016) 122-135. doi:10.1016/j.oceaneng.2015.12.040. 
[25] ITTC, Cavitation committee report, Kobe, Japan, 18th International Towing Tank Conference, October, 1987.

[26] A. Brooker, V. Humphrey, Measurement of radiated underwater noise from a small research vessel in shallow water, Ocean Eng. 120 (2016) 182-189. doi:10.1016/j.oceaneng.2015.09.048.

[27] D. Ross, Mechanics of Underwater Noise, Peninsula Publishing, California, USA, 1976. doi:10.1016/B978-0-08-021182-4.50014-3.

[28] G.P. Haddle, Skudryzyk E. J., The physics of flow noise, J. Acoust. Soc. Am. Rev. Tutor. Pap. Var. Asp. Acoust. 46 (1969) 130-157. doi:10.1121/1.1911663. 
Appendix A. High speed cavitation observation videos

The cavitation observation videos recorded with an underwater camera of the Hydropod during both full-scale trials are provided in this appendix. These recordings are converted to slow motion to provide a clearer understanding.

Click here to download Video: 600RPM_old_propeller.avi

Video 1.: Cavitation observation video with old propeller at 600 RPM

Click here to download Video: 900RPM_old_propeller.avi

Video 2.: Cavitation observation video with old propeller at 900 RPM

Click here to download Video: 1200RPM_old_propeller.avi

Video 3.: Cavitation observation video with old propeller at 1200 RPM

Click here to download Video: 600RPM_NPT_propeller.avi

Video 4.: Cavitation observation video with NPT propeller at 600 RPM

Click here to download Video: 900RPM_NPT_propeller.avi

Video 5.: Cavitation observation video with NPT propeller at 900 RPM

Click here to download Video: 1200RPM_NPT_propeller.avi

Video 6.: Cavitation observation video with NPT propeller at 1200 RPM 
Click here to download Video: 1500RPM_NPT_propeller.avi

Video 7.: Cavitation observation video with NPT propeller at 1500 RPM 